\begin{tabular}{|c|c|}
\hline $\begin{array}{l}\text { Additi } \\
\text { Inform }\end{array}$ & $\begin{array}{l}\text { NOTICE: This is the author's version of a work that was accepted for } \\
\text { publication in Journal of Power Sources. Changes resulting from the } \\
\text { publishing process, such as peer review, editing, corrections, structural } \\
\text { formatting, and other quality control mechanisms may not be reflected in } \\
\text { this document. Changes may have been made to this work since it was } \\
\text { submitted for publication. A definitive version was subsequently } \\
\text { published in Journal of Power Sources, Vol. } 248 \text { (2013). doi: } \\
\text { http://doi.org/10.1016/j.jpowsour.2013.10.050 }\end{array}$ \\
\hline
\end{tabular}


Simultaneous estimation of states and inputs in a planar Solid Oxide Fuel

Cell using nonlinear adaptive observer design

\section{Periasamy Vijay}

Centre for Process Systems Computations, Department of Chemical Engineering, Curtin University, Western Australia 6845, Australia.

Ph: +61 892669890

Email: V.Periasamy@ curtin.edu.au

2. Moses O. Tadé*

Corresponding author

Centre for Process Systems Computations, Department of Chemical Engineering, Curtin University, Western Australia 6845, Australia.

Ph: +61 892667581

Fax: +61 892662681

Email: M.O.Tade@curtin.edu.au

\section{Khaliq Ahmed}

Ceramic Fuel Cells Ltd.,

170 Browns Road, Noble Park, Vic. 3174, Australia.

Email: khaliqa@cfcl.com.au

\section{Ranjeet Utikar}

Centre for Process Systems Computations, Department of Chemical Engineering, Curtin University, Western Australia 6845, Australia.

Ph: +61 892669837

Email: R.Utikar@curtin.edu.au 


\section{Vishnu Pareek}

Centre for Process Systems Computations, Department of Chemical Engineering, Curtin University, Western Australia 6845, Australia.

Ph: +61892664687

Email: V.Pareek@curtin.edu.au 


\title{
Simultaneous estimation of states and inputs in a planar Solid Oxide Fuel Cell using nonlinear adaptive observer design
}

\author{
Periasamy Vijay*, Moses O. Tadé*, Khaliq Ahmed $\dagger$, Ranjeet Utikar*, Vishnu Pareek* \\ *Centre for Process Systems Computations, Department of Chemical Engineering, Curtin \\ University, Western Australia 6845, Australia.
}

†Ceramic Fuel Cells Ltd., 170 Browns Road, Noble Park, Vic. 3174, Australia.

\begin{abstract}
An adaptive nonlinear observer design for the planar solid oxide fuel cell (SOFC) is presented in this work. This observer is based on a lumped parameter model of the SOFC and it can simultaneously estimate the inputs and the states of the system. Considering the inputs as unknown parameters is advantageous because some of the input parameters are not practically measurable in a SOFC stack. The asymptotic stability of the proposed observer is proven using the Lyapunov function method and is based on the concept of input-to-state stability for cascaded systems. The simulations show that the developed observer can track the temperature and species concentration profiles in the planar SOFC during step changes in the input variables and can simultaneously predict the input variables. The adaptive observer presented is valid for a wide operating range, requires fewer variables to be measured, and is robust to fluctuations in the input variables.
\end{abstract}

Keywords: Adaptive non-linear observer; Solid oxide fuel cell; Lyapunov function method; Input and state estimation. 


\section{Introduction}

The challenges in commercializing solid oxide fuel cells (SOFC's) may be classified as those pertaining to material science, reaction kinetics and control and operation. Even if the materials and kinetics related issues are sorted out, there are several technological challenges relating to control and operation that must be addressed before successful commercialisation. One of the important issues in the planar configuration SOFC is the non-uniform spatial temperature distribution in the cell, which places serious limitations on its performance. The non-uniformity of the temperature in the cell will lead to hot spots and thermal stresses that in turn increases the probability of failure and degradation of the cell $[1,2]$. These issues are especially predominant during the transient operation of the SOFC. The thermal gradients are especially high in the case of methane fuelled SOFC with internal reforming. Thermal gradients would also be significant in SOFC systems intended for load following and frequent on/off applications. This thermal management problem has an important bearing on the efficiency, life and reliability of the cell. Therefore, thermal management is essential not only to prevent the damage to the fuel cell and thereby maximise the cell life, but also to improve its efficiency and performance [3, 4]. For effective thermal management, information about the temperature distributions inside the cell is required. Since this is generally not measurable, dynamic estimation is one option for obtaining this information.

The need to minimise the temperature gradients in the cell has been realised in the past and methods have been presented [2] for designing control strategies to minimise the spatial temperature variation in a molten carbonate fuel cell. A numerical bifurcation analysis of the SOFC was performed in [5], which gave guidelines to avoid high over-temperatures in the SOFC. [6, 7] proposed state estimators based on Kalman filter that can estimate the temperature and the concentration profiles inside the molten carbonate fuel cell. In [8], an 
observer was presented for estimating the spatial temperature profiles of a single cell SOFC, which is based on a linearised model. Observers designed using linearization techniques are not very suitable for the SOFC, which is a highly non-linear system.

The limited amount of research in the open literature use linerisation approaches to observer design and consider a single cell design. Many models in the literature consider the cell current as the input $[6,7]$ and treat other inputs to the system like the cathodic and anodic flow rates and temperatures as constant parameters. Some designs consider the inlet mole flow rates as known inputs that are measured [8], which is quite possible if a single cell is considered or if the whole stack is lumped together as a single unit for the design. However, considering the whole stack as single unit may give an unrealistic picture of the temperature distribution within the stack because in a planar SOFC stack, different cells will have different temperature profiles [9] both during steady state and transient operation. If on the other hand, if it is desired to include many cells into the model based observer design, it may not possible to measure the inlet flow rates or the current for each cell. In this situation, it is beneficial to consider the inputs as unknowns and estimate their values in addition to the state variables.

The SOFC system being an inherently nonlinear one requires a nonlinear observer for the purpose of a wider range of applicability and accuracy. Unlike for linear systems, there is no systematic procedure for designing a state observer for a given nonlinear model. Empirical observers and converging observers are the two kinds of observers available for non-linear systems. Kalman filter is a common converging observer which is very useful in the case of linear systems. However, its extension to the nonlinear system, namely the extended Kalman filter is an empirical observer based on some approximation of the nonlinear system or approximation of a theoretical best estimation. It is based on a linearization of the nonlinear 
system in a neighbourhood of its estimation. Hence, the extended Kalman filter is not a globally converging observer. On the other hand theoretically converging observers are only applicable for a certain class of non-linear systems and are usually very difficult to design. Some nonlinear converging observer designs such as sliding mode observers suffer from problems like chattering. Therefore, a Lyapunov function based adaptive observer design has been undertaken, which is a converging observer. Lyapunov function based designs suffer from the problem that there are no systematic methods for finding a suitable Lyapunov function for a given system. However, in this work, the prudent choice of adaptive parameters and the adaptation laws allows the definition of a Lyapunov function, using which the global stability of the error dynamics is proven.

In a previous work by the authors [11], an adaptive nonlinear observer for the planar SOFC was presented. This paper presents an extension of the previous work, in which an unknown input adaptive non-linear observer for the single cell SOFC is presented. It is assumed that the inputs (the cell current density, the anode and the cathode inlet flow rates and the inlet temperature) are not available for measurement and are to be estimated in addition to the system states. It is also assumed that the stack inlet mole fractions (which will be the same for all cells in the stack) and temperature as the measured variables used in the estimation scheme. Similar to the previous work, the asymptotic stability of the proposed observer is proven using the Lyapunov function method considering the system as a cascaded one. The SOFC model used for the observer design is discussed in Section 2. In Section 3, the observer design for the system is presented. In Section 4 the stability analysis is presented, following which, the simulation results are presented in Section 5. Section 6 concludes the paper. 


\section{Modelling}

The SOFC basically consists of three thin layers that constitute the PEN (Positive electrode/Electrolyte/Negative electrode) namely the cathode, the electrolyte and the anode. The PEN is sandwiched between metallic plates called interconnects having channels for gas flow which also serves as current collectors. Hydrogen is supplied on the anode side and oxygen on the cathode side and the electrochemical reaction within the cell produces water vapour on the anode side. The reaction is exothermic and results in the evolution of heat. Figure 1 shows the basic construction and operation of the SOFC. The readers may refer to $[12,13]$ for further details on the construction and functioning of the SOFC.

A lumped parameter model is used to capture the thermal distribution in the cell. The cell model is constructed using 6 lumps for which the mass and the thermal balances are written. Increasing the number of lumps will result in more accurate prediction of the temperature distribution. This model for the hydrogen fed SOFC was presented and validated in [11] and more details can be found there. For the benefit of the reader, the model development is outlined in the appendix.

An improvement in the model used in this work compared to [11] is that the heat capacities of the anode and cathode gases are considered as functions of species mole fractions at the particular lump as given in Eqs. (A17-A20). This makes the observer design more complicated but will improve the accuracy of the model.

In the next section, an unknown input adaptive observer design is proposed for this system. The cell current, the anode and cathode inlet flows and the temperature the inlet streams will be considered as the inputs that need to be estimated in addition to the states. These variables 
will be defined as the adaptive parameters for which adaptation laws will be formulated in such a way so as to ensure the asymptotic stability of the error dynamics.

\section{Adaptive observer design}

In this section, an unknown input adaptive observer based on Lyapunov function approach is presented for estimating the states of the SOFC. It is assumed that the concentrations of the three species at the inlet of the cell are measured and they are same as those in the first lump. Similarly, it is assumed that the temperature of the first lump is measured. The proposed observer equations are,

$$
\begin{aligned}
\rho_{\mathrm{mol}}^{\mathrm{f}} V_{\mathrm{gas}}^{\mathrm{f}} \frac{d \hat{y}_{\mathrm{H}_{2}}^{i}}{d t}= & y_{\mathrm{H}_{2}}^{\mathrm{in}} \hat{F}_{\mathrm{A}}^{\mathrm{in}}-\hat{y}_{\mathrm{H}_{2}}^{i} \hat{F}_{\mathrm{A}}^{\mathrm{in}}-\hat{y}_{\mathrm{H}_{2}}^{i}\left(-\sum_{j=1}^{i} r_{\mathrm{H}_{2}}^{j}(\hat{I})+\sum_{j=1}^{i} r_{\mathrm{H}_{2} \mathrm{O}}^{j}(\hat{I})\right)-r_{\mathrm{H}_{2}}^{i}(\hat{I}) \\
\rho_{\mathrm{mol}}^{\mathrm{f}} V_{\mathrm{gas}}^{\mathrm{f}} \frac{d \hat{y}_{\mathrm{H}_{2}}^{i}}{d t}= & \hat{y}_{\mathrm{H}_{2}}^{i-1} \hat{F}_{\mathrm{A}}^{\mathrm{in}}+\hat{y}_{\mathrm{H}_{2}}^{i-1}\left(-\sum_{j=1}^{i-1} r_{\mathrm{H}_{2}}^{j}(\hat{I})+\sum_{j=1}^{i-1} r_{\mathrm{H}_{2} \mathrm{O}}^{j}(\hat{I})\right)-\hat{y}_{\mathrm{H}_{2}}^{i} \hat{F}_{\mathrm{A}}^{\mathrm{in}} \\
& -\hat{y}_{\mathrm{H}_{2}}^{i}\left(-\sum_{j=1}^{i} r_{\mathrm{H}_{2}}^{j}(\hat{I})+\sum_{j=1}^{i} r_{\mathrm{H}_{2} \mathrm{O}}^{j}(\hat{I})\right)-r_{\mathrm{H}_{2}}^{i}(\hat{I})
\end{aligned}
$$

Where, $i=1$ for Eq. (1) and $i=2, \cdots, 6$ for Eq. (2).

$$
\begin{aligned}
\rho_{\mathrm{mol}}^{\mathrm{f}} V_{\mathrm{gas}}^{\mathrm{f}} \frac{d \hat{y}_{\mathrm{H}_{2} \mathrm{O}}^{i}}{d t}= & y_{\mathrm{H}_{2} \mathrm{O}}^{\text {in }} \hat{F}_{\mathrm{A}}^{\text {in }}-\hat{y}_{\mathrm{H}_{2} \mathrm{O}}^{i} \hat{F}_{\mathrm{A}}^{\text {in }}-\hat{y}_{\mathrm{H}_{2} \mathrm{O}}^{i}\left(-\sum_{j=1}^{i} r_{\mathrm{H}_{2}}^{j}(\hat{I})+\sum_{j=1}^{i} r_{\mathrm{H}_{2} \mathrm{O}}^{j}(\hat{I})\right)+r_{\mathrm{H}_{2} \mathrm{O}}^{i}(\hat{I}) \\
\rho_{\mathrm{mol}}^{\mathrm{f}} V_{\mathrm{gas}}^{\mathrm{f}} \frac{d \hat{y}_{\mathrm{H}_{2} \mathrm{O}}^{i}}{d t}= & \hat{y}_{\mathrm{H}_{2} \mathrm{O}}^{i-1} \hat{F}_{\mathrm{A}}^{\text {in }}+\hat{y}_{\mathrm{H}_{2} \mathrm{O}}^{i-1}\left(-\sum_{j=1}^{i-1} r_{\mathrm{H}_{2}}^{j}(\hat{I})+\sum_{j=1}^{i-1} r_{\mathrm{H}_{2} \mathrm{O}}^{j}(\hat{I})\right)-\hat{y}_{\mathrm{H}_{2} \mathrm{O}}^{i} \hat{F}_{\mathrm{A}}^{\text {in }} \\
& -\hat{y}_{\mathrm{H}_{2} \mathrm{O}}^{i}\left(-\sum_{j=1}^{i} r_{\mathrm{H}_{2}}^{j}(\hat{I})+\sum_{j=1}^{i} r_{\mathrm{H}_{2} \mathrm{O}}^{j}(\hat{I})\right)+r_{\mathrm{H}_{2} \mathrm{O}}^{i}(\hat{I})
\end{aligned}
$$

where, $i=1$ for Eq. (3) and $i=2, \cdots, 6$ for Eq. (4). 


$$
\begin{aligned}
& \rho_{\text {mol }}^{\mathrm{a}} V_{\mathrm{gas}}^{\mathrm{a}} \frac{d \hat{y}_{\mathrm{O}_{2}}^{i}}{d t}=y_{\mathrm{O}_{2}}^{\text {in }} \hat{F}_{\mathrm{C}}^{\text {in }}-\hat{y}_{\mathrm{O}_{2}}^{i} \hat{F}_{\mathrm{C}}^{\text {in }}-\hat{y}_{\mathrm{O}_{2}}^{i}\left(-\sum_{j=1}^{i} r_{\mathrm{O}_{2}}^{j}(\hat{I})\right)-r_{\mathrm{O}_{2}}^{i}(\hat{I}) \\
& \rho_{\mathrm{mol}}^{\mathrm{a}} V_{\mathrm{gas}}^{\mathrm{a}} \frac{d \hat{y}_{\mathrm{O}_{2}}^{i}}{d t}=\hat{y}_{\mathrm{O}_{2}}^{i-1} \hat{F}_{\mathrm{C}}^{\text {in }}+\hat{y}_{\mathrm{O}_{2}}^{i-1}\left(-\sum_{j=1}^{i-1} r_{\mathrm{O}_{2}}^{j}(\hat{I})\right)-\hat{y}_{\mathrm{O}_{2}}^{i} \hat{F}_{\mathrm{C}}^{\text {in }}-\hat{y}_{\mathrm{O}_{2}}^{i}\left(\hat{F}_{\mathrm{C}}^{\text {in }}-\sum_{j=1}^{i} r_{\mathrm{O}_{2}}^{j}(\hat{I})\right)-r_{\mathrm{O}_{2}}^{i}(\hat{I})
\end{aligned}
$$

Where, $i=1$ for Eq. (5) and $i=2, \cdots, 6$ for Eq. (6).

$$
\begin{aligned}
& \rho_{\mathrm{S}} V_{\mathrm{S}} c_{\mathrm{p}}^{\mathrm{s}} \frac{d \hat{T}_{\mathrm{S}}^{i}}{d t}=\left(y_{\mathrm{H}_{2}}^{\mathrm{in}} c_{\mathrm{p}}^{\mathrm{H}_{2}}+y_{\mathrm{H}_{2} \mathrm{O}}^{\mathrm{in}} c_{\mathrm{p}}^{\mathrm{H}_{2} \mathrm{O}}\right) \hat{T}^{\mathrm{in}} \hat{F}_{\mathrm{A}}^{\mathrm{in}}+\left(y_{\mathrm{O}_{2}} c_{\mathrm{p}}^{\mathrm{in}}+y_{\mathrm{N}_{2}}^{\mathrm{in}} c_{\mathrm{p}}^{\mathrm{N}_{2}}\right) \hat{T}^{\mathrm{in}} \hat{F}_{\mathrm{C}}^{\mathrm{in}}-\left(\hat{y}_{\mathrm{H}_{2}}^{i} c_{\mathrm{p}}^{\mathrm{H}_{2}}+\hat{y}_{\mathrm{H}_{2} \mathrm{O}}^{i} c_{\mathrm{p}}^{\mathrm{H}_{2} \mathrm{O}}\right) \hat{T}_{\mathrm{S}}^{i} \hat{F}_{\mathrm{A}}^{\mathrm{in}} \\
&-\left(\hat{y}_{\mathrm{H}_{2}}^{i} c_{\mathrm{p}}^{\mathrm{H}_{2}}+\hat{y}_{\mathrm{H}_{2} \mathrm{O}}^{i} c_{\mathrm{p}}^{\mathrm{H}_{2} \mathrm{O}}\right) \hat{T}_{\mathrm{S}}^{i}\left(-\sum_{j=1}^{i} r_{\mathrm{H}_{2}}^{j}(\hat{I})+\sum_{j=1}^{i} r_{\mathrm{H}_{2} \mathrm{O}}^{j}(\hat{I})\right) \\
&-\left(\hat{y}_{\mathrm{O}_{2}}^{i} c_{\mathrm{p}}^{\mathrm{O}_{2}}+\hat{y}_{\mathrm{N}_{2}}^{\mathrm{in}_{\mathrm{p}}} c_{\mathrm{p}}^{\mathrm{N}_{2}}\right) \hat{T}_{\mathrm{S}}^{i} \hat{F}_{\mathrm{C}}^{\mathrm{in}}-\left(\hat{y}_{\mathrm{O}_{2}}^{i} c_{\mathrm{p}}^{\mathrm{O}_{2}}+\hat{y}_{\mathrm{N}_{2}}^{\mathrm{in}} c_{\mathrm{p}}^{\mathrm{N}_{2}}\right) \hat{T}_{\mathrm{S}}^{i}\left(-\sum_{j=1}^{i} r_{\mathrm{O}_{2}}^{j}(\hat{I})\right)+\frac{\Delta H_{\mathrm{R}} \hat{I}}{2 F} \\
&-\hat{I}\left(C_{E_{0}}^{1}-C_{E_{0}}^{2} \hat{\mathrm{S}}_{\mathrm{S}}^{i}+\frac{R \hat{T}_{\mathrm{S}}^{i}}{2 F} \ln \left(\frac{\hat{y}_{\mathrm{H}_{2}}^{i}\left(\hat{y}_{\mathrm{O}_{2}}^{i}\right)^{0.5}}{\hat{y}_{\mathrm{H}_{2} \mathrm{O}}^{i}}\right)\right) \\
&-\hat{I}\left(-\hat{I} R_{\mathrm{ohm}}-\frac{R \hat{T}_{\mathrm{S}}^{i}}{2 F} \ln \left(\frac{I_{\mathrm{L}}}{I_{\mathrm{L}}-\hat{I}}\right)-\frac{2 R \hat{T}_{\mathrm{S}}^{i}}{F} \sinh ^{-1}\left(\frac{0.5 \hat{I}}{I_{0}}\right)\right) \\
&-E_{T_{\mathrm{S}}^{1}} \\
&\left(-C_{E_{0}}^{2} \hat{I}-\frac{\hat{I} R}{2 F} \ln \left(\frac{I_{\mathrm{L}}}{I_{\mathrm{L}}-\hat{I}}\right)\right. \\
&\left.-\frac{2 \hat{I} R}{F} \sinh ^{-1}\left(\frac{0.5 \hat{I}}{I_{0}}\right)+\frac{\hat{I} R}{2 F} \ln \left(\frac{\left(\hat{y}_{\mathrm{H}_{2}}^{1}\right)\left(\hat{y}_{\mathrm{O}_{2}}^{1}\right)^{0.5}}{\left(\hat{y}_{\mathrm{H}_{2} \mathrm{O}}^{1}\right)}\right)\right)
\end{aligned}
$$




$$
\begin{aligned}
\rho_{\mathrm{S}} V_{\mathrm{S}} c_{\mathrm{p}}^{\mathrm{S}} \frac{d \hat{T}_{\mathrm{S}}^{i}}{d t}= & \left(\hat{y}_{\mathrm{H}_{2}}^{i} c_{\mathrm{p}}^{\mathrm{H}_{2}}+\hat{y}_{\mathrm{H}_{2} \mathrm{O}}^{i} c_{\mathrm{p}}^{\mathrm{H}_{2} \mathrm{O}}\right) \hat{T}_{\mathrm{S}}^{i-1} \hat{F}_{\mathrm{A}}^{\mathrm{in}}+\left(\hat{y}_{\mathrm{H}_{2}}^{i} c_{\mathrm{p}}^{\mathrm{H}_{2}}+\hat{y}_{\mathrm{H}_{2} \mathrm{O}}^{i} c_{\mathrm{p}}^{\mathrm{H}_{2} \mathrm{O}}\right) \hat{T}_{\mathrm{S}}^{i-1}\left(-\sum_{j=1}^{i-1} r_{\mathrm{H}_{2}}^{j}(\hat{I})+\sum_{j=1}^{i-1} r_{\mathrm{H}_{2} \mathrm{O}}^{j}(\hat{I})\right) \\
& +\left(\hat{y}_{\mathrm{O}_{2}}^{i} c_{\mathrm{p}}^{\mathrm{O}_{2}}+y_{\mathrm{N}_{2}}^{\text {in }} c_{\mathrm{p}}^{\mathrm{N}_{2}}\right) \hat{T}_{\mathrm{S}}^{i-1} \hat{F}_{\mathrm{C}}^{\mathrm{in}}+\left(\hat{y}_{\mathrm{O}_{2}}^{i} c_{\mathrm{p}}^{\mathrm{O}_{2}}+y_{\mathrm{N}_{2}}^{\mathrm{in}} c_{\mathrm{p}}^{\mathrm{N}_{2}}\right) \hat{T}_{\mathrm{S}}^{i-1}\left(-\sum_{j=1}^{i-1} r_{\mathrm{O}_{2}}^{j}(\hat{I})\right)-\left(\hat{y}_{\mathrm{H}_{2}}^{i} c_{\mathrm{p}}^{\mathrm{H}_{2}}+\hat{y}_{\mathrm{H}_{2} \mathrm{O}}^{i} c_{\mathrm{p}}^{\mathrm{H}_{2} \mathrm{O}}\right) \hat{T}_{\mathrm{S}}^{i} \hat{F}_{\mathrm{A}}^{\mathrm{in}} \\
& -\left(\hat{y}_{\mathrm{O}_{2}}^{i} c_{\mathrm{p}}^{\mathrm{O}_{2}}+y_{\mathrm{N}_{2}}^{\mathrm{in}} c_{\mathrm{p}}^{\mathrm{N}_{2}}\right) \hat{T}_{\mathrm{S}}^{i} \hat{F}_{\mathrm{C}}^{\mathrm{in}}-\left(\hat{y}_{\mathrm{H}_{2}}^{i} c_{\mathrm{p}}^{\mathrm{H}_{2}}+\hat{y}_{\mathrm{H}_{2} \mathrm{O}}^{i} c_{\mathrm{p}}^{\mathrm{H}_{2} \mathrm{O}}\right) \hat{T}_{\mathrm{S}}^{i}\left(-\sum_{j=1}^{i} r_{\mathrm{H}_{2}}^{j}(\hat{I})+\sum_{j=1}^{i} r_{\mathrm{H}_{2} \mathrm{O}}^{j}(\hat{I})\right) \\
& -\left(\hat{y}_{\mathrm{O}_{2}}^{i} c_{\mathrm{p}}^{\mathrm{O}_{2}}+y_{\mathrm{N}_{2}} c_{\mathrm{p}}^{\mathrm{in}}\right) \hat{T}_{\mathrm{S}}^{i}\left(-\sum_{j=1}^{i} r_{\mathrm{O}_{2}}^{j}(\hat{I})\right)+\frac{\Delta H_{\mathrm{R}} \hat{I}}{2 F} \\
& -\hat{I}\left(C_{E_{0}}^{1}-C_{E_{0}}^{2} \hat{T}_{\mathrm{S}}^{i}+\frac{R \hat{T}_{\mathrm{S}}^{i}}{2 F} \ln \left(\frac{\hat{y}_{\mathrm{H}_{2}}^{i}\left(\hat{y}_{\mathrm{O}_{2}}^{i}\right)^{0.5}}{\hat{y}_{\mathrm{H}_{2} \mathrm{O}}^{i}}\right)\right) \\
& -\hat{I}\left(-\hat{I} R_{\mathrm{ohm}}-\frac{R \hat{T}_{\mathrm{S}}^{i}}{2 F} \ln \left(\frac{I_{\mathrm{L}}}{I_{\mathrm{L}}-\hat{I}}\right)-\frac{2 R \hat{T}_{\mathrm{S}}^{i}}{F} \sinh ^{-1}\left(\frac{0.5 \hat{I}}{I_{0}}\right)\right)
\end{aligned}
$$

where, $i=1$ for Eq. (7) and $i=2, \cdots, 6$ for Eq. (8). The parameter adaptation laws are given as:

$$
\begin{aligned}
& \frac{d \hat{F}_{\mathrm{A}}^{\text {in }}}{d t}=\frac{\lambda_{1}}{\rho_{\text {mol }}^{\mathrm{f}} V_{\mathrm{gas}}^{\mathrm{f}}}\left(+E_{y_{\mathrm{H}_{2}}^{1}} y_{\mathrm{H}_{2}}^{\text {in }}-\hat{y}_{\mathrm{H}_{2}}^{1} E_{y_{\mathrm{H}_{2}}^{1}}+E_{y_{\mathrm{H}_{2} \mathrm{O}}^{1}} y_{\mathrm{H}_{2} \mathrm{O}}^{\text {in }}-\hat{y}_{\mathrm{H}_{2} \mathrm{O}}^{1} E_{y_{\mathrm{H}_{2} \mathrm{O}}^{1}}\right) \\
& \frac{d \hat{F}_{\mathrm{C}}^{\text {in }}}{d t}=\frac{\lambda_{2}}{\rho_{\mathrm{mol}}^{\mathrm{a}} V_{\mathrm{gas}}^{\mathrm{a}}}\left(+E_{y_{\mathrm{O}_{2}}^{1}} y_{\mathrm{O}_{2}}^{\text {in }}-\hat{y}_{\mathrm{O}_{2}}^{1} E_{y_{\mathrm{O}_{2}}}\right)
\end{aligned}
$$

$$
\begin{aligned}
& \frac{d \hat{I}}{d t}=\frac{\lambda_{3}}{\rho_{\text {mol }}^{\mathrm{a}} V_{\mathrm{gas}}^{\mathrm{a}}}\left(\begin{array}{l}
-\hat{y}_{\mathrm{H}_{2}}^{1} E_{y_{\mathrm{H}_{2}}^{1}} \frac{\left(v_{\mathrm{H}_{2} \mathrm{O}}-v_{\mathrm{H}_{2}}\right) S}{2 F}-E_{y_{\mathrm{H}_{2}}^{1}} \frac{v_{\mathrm{H}_{2}} S}{2 F} \\
-\hat{y}_{\mathrm{H}_{2} \mathrm{O}}^{1} E_{y_{\mathrm{H}_{2} \mathrm{O}}^{1}} \frac{\left(v_{\mathrm{H}_{2} \mathrm{O}}-v_{\mathrm{H}_{2}}\right) S}{2 F}+E_{y_{\mathrm{H}_{2} \mathrm{O}}^{1}} \frac{v_{\mathrm{H}_{2} \mathrm{O}} S}{2 F}
\end{array}\right) \\
& +\frac{1}{\rho_{\text {mol }}^{\mathrm{c}} V_{\text {gas }}^{\mathrm{c}}}\left(\hat{y}_{\mathrm{O}_{2}}^{1} E_{y_{\mathrm{O}_{2}}^{1}} \frac{v_{\mathrm{O}_{2}} S}{2 F}-E_{y_{\mathrm{O}_{2}}^{1}} \frac{v_{\mathrm{O}_{2}} S}{2 F}\right)
\end{aligned}
$$


$\frac{d \hat{T}^{\mathrm{in}}}{d t}=\frac{\lambda_{4}}{\rho_{\mathrm{S}} V_{\mathrm{S}} c_{\mathrm{p}}^{\mathrm{S}}}\left(\hat{F}_{\mathrm{A}}^{\text {in }}\left(y_{\mathrm{H}_{2}}^{\mathrm{in}} c_{\mathrm{p}}^{\mathrm{H}_{2}}+y_{\mathrm{H}_{2} \mathrm{O}}^{\mathrm{in}} c_{\mathrm{p}}^{\mathrm{H}_{2} \mathrm{O}}\right) E_{T_{\mathrm{S}}^{1}}+\hat{F}_{\mathrm{C}}^{\text {in }}\left(y_{\mathrm{O}_{2}}^{\text {in }} c_{\mathrm{p}}^{\mathrm{O}_{2}}+y_{\mathrm{N}_{2}}^{\mathrm{in}} c_{\mathrm{p}}^{\mathrm{N}_{2}}\right) E_{T_{\mathrm{S}}^{1}}\right)$

where $\lambda_{1}, \lambda_{2}, \lambda_{3}, \lambda_{4}>0$. The $\lambda$ 's in the adaptation laws are the tuning parameters introduced to influence the convergence time for the estimation, since only the asymptotic stability of the error dynamics is assured. Therefore, the tuning parameters will help to achieve faster settling time. This can be understood as being analogous to decreasing the capacitance of a thermal or electrical system, thereby achieving a decreased time constant. The adaptation laws are designed in such a way that the error dynamics become asymptotically stable as described in the next section.

\section{Stability of the error dynamics}

In this section, the stability analysis for the error dynamics is presented using the Lyapunov function method. For the purpose of proving the asymptotic stability of the system, the parameter and state estimation errors are classified into the following groups:

$$
\begin{aligned}
& \mathbf{E}_{1}=\left[\begin{array}{llllll}
E_{F_{\mathrm{A}}^{\text {in }}} & E_{F_{\mathrm{C}}^{\mathrm{in}}} & E_{y_{\mathrm{H}_{2}}^{1}} & E_{y_{\mathrm{H}_{2} \mathrm{O}}^{1}} & E_{y_{\mathrm{O}_{2}}^{1}} & E_{I}
\end{array}\right]^{T}, \\
& \mathbf{E}_{2}=\left[\begin{array}{lll}
E_{y_{\mathrm{H}_{2}}^{i}} & E_{y_{\mathrm{H}_{2} \mathrm{O}}^{i}} & E_{y_{\mathrm{O}_{2}}^{i}}
\end{array}\right]^{T} \text {, where } i=2, \cdots, 6 \text {. } \\
& \mathbf{E}_{3}=\left[\begin{array}{ll}
E_{T_{\mathrm{S}}^{1}} & E_{T^{\text {in }}}
\end{array}\right]^{T} \\
& \mathbf{E}_{4}=\left[E_{T_{\mathrm{S}}^{i}}\right]^{T} \text {, where } i=2, \ldots, 6 \text {. }
\end{aligned}
$$


where $E_{F_{\mathrm{A}}^{\text {in }}}, E_{F_{\mathrm{C}}^{\text {in }}}, E_{y_{\mathrm{H}_{2}}^{i}} E_{y_{\mathrm{H}_{2} \mathrm{O}}^{i}} E_{y_{\mathrm{O}_{2}}^{i}}, E_{T_{\mathrm{S}}^{i}}, E_{I}, E_{T^{\text {in }}}$ are the parameter and the state estimation errors given as:

$$
\begin{aligned}
& E_{F_{\mathrm{A}}^{\text {in }}}=F_{\mathrm{A}}^{\text {in }}-\hat{F}_{\mathrm{A}}^{\text {in }} \\
& E_{F_{\mathrm{C}}^{\text {in }}}=F_{\mathrm{C}}^{\text {in }}-\hat{F}_{\mathrm{C}}^{\text {in }}
\end{aligned}
$$

$$
E_{y_{\mathrm{H}_{2}}^{i}}=y_{\mathrm{H}_{2}}^{i}-\hat{y}_{\mathrm{H}_{2}}^{i}
$$

$$
E_{y_{\mathrm{H}_{2} \mathrm{O}}^{i}}=y_{\mathrm{H}_{2} \mathrm{O}}^{i}-\hat{y}_{\mathrm{H}_{2} \mathrm{O}}^{i}
$$

$$
E_{y_{\mathrm{O}_{2}}^{i}}=y_{\mathrm{O}_{2}}^{i}-\hat{y}_{\mathrm{O}_{2}}^{i}
$$

$$
E_{T_{\mathrm{S}}^{i}}=T_{\mathrm{S}}^{i}-\hat{T}_{\mathrm{S}}^{i}
$$

$$
E_{I}=I-\hat{I}
$$

$$
E_{T^{\text {in }}}=T^{\text {in }}-\hat{T}^{\text {in }}
$$

From model equations in Eqs. (A21-A28) and observer equations in Eqs. (1-12), the error dynamics are derived as follows:

$$
\begin{aligned}
\rho_{\mathrm{mol}}^{\mathrm{f}} V_{\mathrm{gas}}^{\mathrm{f}} \frac{d E_{y_{\mathrm{H}_{2}}^{i}}}{d t}= & E_{F_{\mathrm{A}}} y_{\mathrm{H}_{2}}^{\text {in }}-E_{y_{\mathrm{H}_{2}}^{i}}\left(F_{\mathrm{A}}^{\mathrm{in}}+\frac{\left(v_{\mathrm{H}_{2} \mathrm{O}}-v_{\mathrm{H}_{2}}\right) S}{2 F}(i) I\right)-\hat{y}_{\mathrm{H}_{2}}^{i} E_{F_{\mathrm{A}}^{\text {in }}} \\
& -\hat{y}_{\mathrm{H}_{2}}^{i} E_{I} \frac{\left(v_{\mathrm{H}_{2} \mathrm{O}}-v_{\mathrm{H}_{2}}\right) S}{2 F}(i)-E_{I} \frac{\left(v_{\mathrm{H}_{2}}\right) S}{2 F}(i)
\end{aligned}
$$




$$
\begin{gathered}
\rho_{\mathrm{mol}}^{\mathrm{f}} V_{\mathrm{gas}^{\mathrm{fas}}}^{\mathrm{f}} \frac{d E_{y_{\mathrm{H}_{2}}^{i}}}{d t}=E_{y_{\mathrm{H}_{2}}}\left(F_{\mathrm{A}}^{\text {in }}+\frac{\left(v_{\mathrm{H}_{2} \mathrm{O}}-v_{\mathrm{H}_{2}}\right) S}{2 F}(i-1) I\right)+\hat{y}_{\mathrm{H}_{2}}^{i-1} E_{F_{\mathrm{A}}^{\mathrm{in}}}+\hat{y}_{\mathrm{H}_{2}}^{i-1} E_{I} \frac{\left(v_{\mathrm{H}_{2} \mathrm{O}}-v_{\mathrm{H}_{2}}\right) S}{2 F}(i-1) \\
-E_{y_{\mathrm{H}_{2}}^{i}}\left(F_{\mathrm{A}}^{\text {in }}+\frac{\left(v_{\mathrm{H}_{2} \mathrm{O}}-v_{\mathrm{H}_{2}}\right) S}{2 F}(i) I\right)-\hat{y}_{\mathrm{H}_{2}}^{i} E_{F_{\mathrm{A}}^{\mathrm{in}}}-\hat{y}_{\mathrm{H}_{2}}^{i} E_{I} \frac{\left(v_{\mathrm{H}_{2} \mathrm{O}}-v_{\mathrm{H}_{2}}\right) S}{2 F}(i)-E_{I} \frac{\left(v_{\mathrm{H}_{2}}\right) S}{2 F}(i)
\end{gathered}
$$

Where, $i=1$ for Eq. (25) and $i=2, \cdots, 6$ for Eq. (26).

$$
\begin{aligned}
& \rho_{\mathrm{mol}}^{\mathrm{f}} V_{\mathrm{gas}}^{\mathrm{f}} \frac{d E_{\mathrm{H}_{2} \mathrm{O}}^{i}}{d t}= E_{F_{\mathrm{A}}^{\mathrm{in}}} y_{\mathrm{H}_{2} \mathrm{O}}^{\text {in }}-E_{y_{\mathrm{H}_{2} \mathrm{O}}^{i}}\left(F_{\mathrm{A}}^{\mathrm{in}}+\frac{\left(v_{\mathrm{H}_{2} \mathrm{O}}-v_{\mathrm{H}_{2}}\right) S}{2 F}(i) I\right)-\hat{y}_{\mathrm{H}_{\mathrm{H}_{2} \mathrm{O}}}^{i} E_{F_{\mathrm{A}}^{\mathrm{in}}} \\
&-\hat{y}_{\mathrm{H}_{2} \mathrm{O}}^{i} E_{I} \frac{\left(v_{\mathrm{H}_{2} \mathrm{O}}-v_{\mathrm{H}_{2}}\right) S}{2 F}(i)+E_{I} \frac{\left(v_{\mathrm{H}_{2} \mathrm{O}}\right) S}{2 F}(i) \\
& \rho_{\mathrm{mol}}^{\mathrm{f}} V_{\mathrm{gas}}^{\mathrm{f}} \frac{d E_{y_{\mathrm{H}_{2} \mathrm{O}}^{i}}^{i}}{d t}=E_{y_{\mathrm{H}_{2} \mathrm{O}}^{i-}}\left(F_{\mathrm{A}}^{\mathrm{in}}+\frac{\left(v_{\mathrm{H}_{2} \mathrm{O}}-v_{\mathrm{H}_{2}}\right) S}{2 F}(i-1) I\right)+\hat{y}_{\mathrm{H}_{2} \mathrm{O}}^{i-1} E_{F_{\mathrm{A}}}^{\text {in }}+\hat{y}_{\mathrm{H}_{2}}^{i-1} E_{I} \frac{\left(v_{\mathrm{H}_{2} \mathrm{O}}-v_{\mathrm{H}_{2}}\right) S}{2 F}(i-1) \\
&-E_{y_{\mathrm{H}_{2} \mathrm{O}}^{i}}\left(F_{\mathrm{A}}^{\text {in }}+\frac{\left(v_{\mathrm{H}_{2} \mathrm{O}}-v_{\mathrm{H}_{2}}\right) S}{2 F}(i) I\right)-\hat{y}_{\mathrm{H}_{2} \mathrm{O}}^{i} E_{F_{\mathrm{A}}}-\hat{y}_{\mathrm{H}_{2} \mathrm{O}}^{i} E_{I} \frac{\left(v_{\mathrm{H}_{2} \mathrm{O}}-v_{\mathrm{H}_{2}}\right) S}{2 F}(i)+E_{I} \frac{\left(v_{\mathrm{H}_{2} \mathrm{O}}\right) S}{2 F}(i)
\end{aligned}
$$

Where, $i=1$ for Eq. (27) and $i=2, \cdots, 6$ for Eq. (28).

$$
\begin{gathered}
\rho_{\text {mol }}^{\mathrm{a}} V_{\mathrm{gas}}^{\mathrm{a}} \frac{d E_{y_{\mathrm{O}_{2}}^{i}}}{d t}=E_{F_{\mathrm{C}}^{\mathrm{in}}} y_{\mathrm{O}_{2}}^{\text {in }}-E_{y_{\mathrm{O}_{2}}^{i}}\left(F_{\mathrm{C}}^{\text {in }}-\frac{v_{\mathrm{O}_{2}} S}{2 F}(i) I\right)-\hat{y}_{\mathrm{O}_{2}}^{i} E_{F_{\mathrm{C}}^{\mathrm{in}}}+\hat{y}_{\mathrm{O}_{2}}^{i} E_{I} \frac{v_{\mathrm{O}_{2}} S}{2 F}(i)-E_{I} \frac{v_{\mathrm{O}_{2}} S}{2 F}(i) \\
\rho_{\text {mol }}^{\mathrm{a}} V_{\mathrm{gas}}^{\mathrm{a}} \frac{d E_{y_{\mathrm{O}_{2}}^{i}}^{i}}{d t}=E_{y_{\mathrm{O}_{2}}}\left(F_{\mathrm{C}}^{\mathrm{in}}-\frac{v_{\mathrm{O}_{2}} S}{2 F}(i-1) I\right)+\hat{y}_{\mathrm{O}_{2}}^{i-1} E_{F_{\mathrm{C}}}^{\text {in }}-\hat{y}_{\mathrm{O}_{2}}^{i-1} E_{I} \frac{v_{\mathrm{O}_{2}} S}{2 F}(i-1) \\
-E_{y_{\mathrm{O}_{2}}^{i}}\left(F_{\mathrm{C}}^{\text {in }}-\frac{v_{\mathrm{O}_{2}} S}{2 F}(i) I\right)-\hat{y}_{\mathrm{O}_{2}}^{i} E_{F_{\mathrm{C}}^{\text {in }}}+\hat{y}_{\mathrm{O}_{2}}^{i} E_{I} \frac{v_{\mathrm{O}_{2}} S}{2 F}-E_{I} \frac{v_{\mathrm{O}_{2}} S}{2 F}(i)
\end{gathered}
$$

Where, $i=1$ for Eq. (29) and $i=2, \cdots, 6$ for Eq. (30). 


$$
\begin{aligned}
& \rho_{\mathrm{S}} V_{\mathrm{S}} c_{\mathrm{p}}^{\mathrm{S}} \frac{d E_{T_{\mathrm{S}}^{i}}}{d t}=T^{\mathrm{in}} E_{F_{\mathrm{A}}^{\text {in }}}\left(-y_{\mathrm{H}_{2}}^{\text {in }} c_{\mathrm{p}}^{\mathrm{H}_{2}}-y_{\mathrm{H}_{2} \mathrm{O}}^{\text {in }} c_{\mathrm{p}}^{\mathrm{H}_{2} \mathrm{O}}\right)+\hat{F}_{\mathrm{A}}^{\text {in }} E_{T_{\mathrm{A}}^{\text {in }}}\left(y_{\mathrm{H}_{2}}^{\text {in }} c_{\mathrm{p}}^{\mathrm{H}_{2}}+y_{\mathrm{H}_{2} \mathrm{O}}^{\mathrm{in}} c_{\mathrm{p}}^{\mathrm{H}_{2} \mathrm{O}}\right) \\
& -\left(y_{\mathrm{O}_{2}}^{\text {in }} c_{\mathrm{p}}^{\mathrm{O}_{2}}+y_{\mathrm{N}_{2}}^{\text {in }} c_{\mathrm{p}}^{\mathrm{N}_{2}}\right) T^{\text {in }} E_{F_{\mathrm{C}}^{\text {in }}}+\left(y_{\mathrm{O}_{2}}^{\text {in }} c_{\mathrm{p}}^{\mathrm{O}_{2}}+y_{\mathrm{N}_{2}}^{\text {in }} c_{\mathrm{p}}^{\mathrm{N}_{2}}\right) \hat{F}_{\mathrm{C}}^{\text {in }} E_{T_{\mathrm{C}}^{\text {in }}} \\
& -E_{y_{\mathrm{H}_{2}}}\left(c_{\mathrm{p}}^{\mathrm{H}_{2}} \hat{T}_{\mathrm{S}}^{i} \hat{F}_{\mathrm{A}}^{\mathrm{in}}+c_{\mathrm{p}}^{\mathrm{H}_{2}} \hat{T}_{\mathrm{S}}^{i} E_{F_{\mathrm{A}}}+c_{\mathrm{p}}^{\mathrm{H}_{2}} T_{\mathrm{S}}^{i} \frac{\left(v_{\mathrm{H}_{2} \mathrm{O}}-v_{\mathrm{H}_{2}}\right) S}{2 F}(i) \hat{I}+c_{\mathrm{p}}^{\mathrm{H}_{2}} T_{\mathrm{S}}^{i} \frac{\left(v_{\mathrm{H}_{2} \mathrm{O}}-v_{\mathrm{H}_{2}}\right) S}{2 F}(i) E_{I}\right) \\
& -E_{y_{\mathrm{H}_{2} \mathrm{O}}^{i}}\left(c_{\mathrm{p}}^{\mathrm{H}_{2} \mathrm{O}} \hat{T}_{\mathrm{S}}^{i} \hat{F}_{\mathrm{A}}^{\mathrm{in}}+c_{\mathrm{p}}^{\mathrm{H}_{2} \mathrm{O}} \hat{T}_{\mathrm{S}}^{i} E_{F_{\mathrm{A}}}^{\text {in }}+c_{\mathrm{p}}^{\mathrm{H}_{2} \mathrm{O}} T_{\mathrm{S}}^{i} \frac{\left(v_{\mathrm{H}_{2} \mathrm{O}}-v_{\mathrm{H}_{2}}\right) S}{2 F}(i) \hat{I}+c_{\mathrm{p}}^{\mathrm{H}_{2} \mathrm{O}} T_{\mathrm{S}}^{i} \frac{\left(v_{\mathrm{H}_{2} \mathrm{O}}-v_{\mathrm{H}_{2}}\right) S}{2 F}(i) E_{I}\right) \\
& -E_{y_{\mathrm{O}_{2}}^{i}}\left(c_{\mathrm{p}}^{\mathrm{O}_{2}} \hat{T}_{\mathrm{S}}^{i} \hat{F}_{\mathrm{C}}^{\mathrm{in}}+c_{\mathrm{p}}^{\mathrm{O}_{2}} \hat{T}_{\mathrm{S}}^{i} E_{F_{\mathrm{C}}}-c_{\mathrm{p}}^{\mathrm{O}_{2}} T_{\mathrm{S}}^{i} \frac{v_{\mathrm{O}_{2}} S}{2 F}(i) \hat{I}-c_{\mathrm{p}}^{\mathrm{O}_{2}} T_{\mathrm{S}}^{i} \frac{v_{\mathrm{O}_{2}} S}{2 F}(i) E_{I}\right) \\
& -E_{F_{\mathrm{A}}^{\text {in }}}\left(\hat{y}_{\mathrm{H}_{2}}^{i} c_{\mathrm{p}}^{\mathrm{H}_{2}} \hat{T}_{\mathrm{S}}^{i}+\hat{y}_{\mathrm{H}_{2} \mathrm{O}}^{\mathrm{i}} c_{\mathrm{p}}^{\mathrm{H}_{2} \mathrm{O}} \hat{T}_{\mathrm{S}}^{i}\right)-E_{F_{\mathrm{C}}^{\text {in }}}\left(\hat{y}_{\mathrm{O}_{2}}^{i} c_{\mathrm{p}}^{\mathrm{O}_{2}} \hat{T}_{\mathrm{S}}^{i}+y_{\mathrm{N}_{2}}^{\mathrm{i}} c_{\mathrm{p}}^{\mathrm{N}_{2}} T_{\mathrm{S}}^{i}\right) \\
& -E_{\mathrm{T}_{\mathrm{S}}^{i}}\left(\begin{array}{l}
\hat{y}_{\mathrm{H}_{2}}^{i} c_{\mathrm{p}}^{\mathrm{H}_{2}} \hat{F}_{\mathrm{A}}^{\mathrm{in}}+\hat{y}_{\mathrm{H}_{2} \mathrm{O}}^{i} c_{\mathrm{p}}^{\mathrm{H}_{2} \mathrm{O}} \hat{F}_{\mathrm{A}}^{\mathrm{in}}+\hat{y}_{\mathrm{H}_{2}}^{i} c_{\mathrm{p}}^{\mathrm{H}_{2}} \frac{\left(v_{\mathrm{H}_{2} \mathrm{O}}-v_{\mathrm{H}_{2}}\right) S}{2 F}(i) \hat{I}+\hat{y}_{\mathrm{H}_{2} \mathrm{O}}^{i} c_{\mathrm{p}}^{\mathrm{H}_{2} \mathrm{O}} \frac{\left(v_{\mathrm{H}_{2} \mathrm{O}}-v_{\mathrm{H}_{2}}\right) S}{2 F}(i) \hat{I} \\
+\hat{y}_{\mathrm{O}_{2}}^{i} c_{\mathrm{p}}^{\mathrm{O}_{2}} \hat{F}_{\mathrm{C}}^{\mathrm{in}}-\hat{y}_{\mathrm{O}_{2}}^{i} c_{\mathrm{p}}^{\mathrm{O}_{2}} \frac{v_{\mathrm{O}_{2}} S}{2 F}(i) \hat{I}+y_{\mathrm{N}_{2}}^{\text {in }} c_{\mathrm{p}}^{\mathrm{N}_{2}} \frac{v_{\mathrm{O}_{2}} S}{2 F}(i) \hat{I}+y_{\mathrm{N}_{2}}^{\text {in }} c_{\mathrm{p}}^{\mathrm{N}_{2}} \hat{F}_{\mathrm{C}}^{\text {in }}-C_{E_{0}}^{2} \hat{I}
\end{array}\right) \\
& -E_{I}\left(\begin{array}{l}
\hat{y}_{\mathrm{H}_{2}}^{i} c_{\mathrm{p}}^{\mathrm{H}_{2}} \hat{T}_{\mathrm{S}}^{i} \frac{\left(v_{\mathrm{H}_{2} \mathrm{O}}-v_{\mathrm{H}_{2}}\right) S}{2 F}(i)+\hat{y}_{\mathrm{H}_{2} \mathrm{O}}^{i} c_{\mathrm{p}}^{\mathrm{H}_{2} \mathrm{O}} \hat{T}_{\mathrm{S}}^{i} \frac{\left(v_{\mathrm{H}_{2} \mathrm{O}}-v_{\mathrm{H}_{2}}\right) S}{2 F}-\hat{y}_{\mathrm{O}_{2}}^{i} c_{\mathrm{p}}^{\mathrm{O}_{2}} \hat{T}_{\mathrm{S}}^{i} \frac{v_{\mathrm{O}_{2}} S}{2 F}(i) \\
-y_{\mathrm{N}_{2}}^{\mathrm{in}} c_{\mathrm{p}}^{\mathrm{N}_{2}} T_{\mathrm{S}}^{i} \frac{v_{\mathrm{O}_{2}} S}{2 F}(i)-\frac{\Delta H_{\mathrm{R}}}{2 F}+C_{E_{0}}^{1}-C_{E_{0}}^{2} T_{\mathrm{S}}^{i}+(I+\hat{I}) R_{\mathrm{ohm}}
\end{array}\right) \\
& +\frac{\left(E_{I}+\hat{I}\right) R T_{\mathrm{S}}^{i}}{2 F} \ln \left(\frac{I_{\mathrm{L}}}{I_{\mathrm{L}}-E_{I}-\hat{I}}\right)-\frac{\hat{I} R \hat{T}_{\mathrm{S}}^{i}}{2 F} \ln \left(\frac{I_{\mathrm{L}}}{I_{\mathrm{L}}-\hat{I}}\right)+\frac{2\left(E_{I}+\hat{I}\right) T_{\mathrm{S}}^{i} R}{F} \sinh ^{-1}\left(\frac{0.5\left(E_{I}+\hat{I}\right)}{I_{0}}\right) \\
& -\frac{2 \hat{I} T_{\mathrm{S}}^{i} R}{F} \sinh ^{-1}\left(\frac{0.5 \hat{I}}{I_{0}}\right)-\frac{\left(E_{I}+\hat{I}\right) R T_{\mathrm{S}}^{i}}{2 F} \ln \left(\frac{\left(E_{y_{\mathrm{H}_{2}}^{i}}+\hat{y}_{\mathrm{H}_{2}}^{i}\right)\left(E_{y_{\mathrm{O}_{2}}^{i}}+\hat{y}_{\mathrm{O}_{2}}^{i}\right)^{0.5}}{\left(E_{\mathrm{H}_{\mathrm{H}^{\mathrm{O}}}}+\hat{y}_{\mathrm{H}_{2} \mathrm{O}}^{i}\right)}\right)+\frac{\hat{I} R \hat{T}_{\mathrm{S}}^{i}}{2 F} \ln \left(\frac{\left(\hat{y}_{\mathrm{H}_{2}}^{i}\right)\left(\hat{y}_{\mathrm{O}_{2}}^{i}\right)^{0.5}}{\left(\hat{y}_{\mathrm{H}_{2} \mathrm{O}}^{i}\right)}\right)
\end{aligned}
$$




$$
\begin{aligned}
& \rho_{\mathrm{S}} V_{\mathrm{S}} c_{\mathrm{p}}^{\mathrm{s}} \frac{d E_{T_{\mathrm{S}}^{i}}}{d t}=E_{T_{\mathrm{s}}^{i-1}}\left(\begin{array}{l}
\hat{y}_{\mathrm{H}_{2}}^{i} c_{\mathrm{p}}^{\mathrm{H}_{2}} \hat{F}_{\mathrm{A}}^{\mathrm{in}}+\hat{y}_{\mathrm{H}_{2} \mathrm{O}}^{i} c_{\mathrm{p}}^{\mathrm{H}_{2} \mathrm{O}} \hat{F}_{\mathrm{A}}^{\mathrm{in}}+\hat{y}_{\mathrm{H}_{2}}^{i} c_{\mathrm{p}}^{\mathrm{H}_{2}} \frac{\left(v_{\mathrm{H}_{2} \mathrm{O}}-v_{\mathrm{H}_{2}}\right) S}{2 F}(i) \hat{I}+\hat{y}_{\mathrm{H}_{2} \mathrm{O}}^{i} c_{\mathrm{p}}^{\mathrm{H}_{2} \mathrm{O}} \frac{\left(v_{\mathrm{H}_{2} \mathrm{O}}-v_{\mathrm{H}_{2}}\right) S}{2 F}(i) \hat{I} \\
+\hat{y}_{\mathrm{O}_{2}}^{i} c_{\mathrm{p}}^{\mathrm{O}_{2}} \hat{F}_{\mathrm{C}}^{\text {in }}-\hat{y}_{\mathrm{O}_{2}}^{i} c_{\mathrm{p}}^{\mathrm{O}_{2}} \frac{v_{\mathrm{O}_{2}} S}{2 F}(i) \hat{I}+y_{\mathrm{N}_{2}}^{\text {in }} c_{\mathrm{p}}^{\mathrm{N}_{2}} \frac{v_{\mathrm{O}_{2}} S}{2 F}(i) \hat{I}+y_{\mathrm{N}_{2}}^{\text {in }} c_{\mathrm{p}}^{\mathrm{N}_{2}} \hat{F}_{\mathrm{C}}^{\text {in }}
\end{array}\right) \\
& -E_{y_{\mathrm{H}_{2}}}\left(\begin{array}{l}
c_{\mathrm{p}}^{\mathrm{H}_{2}} \hat{T}_{\mathrm{S}}^{i} \hat{F}_{\mathrm{A}}^{\mathrm{in}}+c_{\mathrm{p}}^{\mathrm{H}_{2}} \hat{T}_{\mathrm{S}}^{i} E_{F_{\mathrm{A}}^{\mathrm{in}}}+c_{\mathrm{p}}^{\mathrm{H}_{2}} T_{\mathrm{S}}^{i} \frac{\left(v_{\mathrm{H}_{2} \mathrm{O}}-v_{\mathrm{H}_{2}}\right) S}{2 F}(i) \hat{I}+c_{\mathrm{p}}^{\mathrm{H}_{2}} T_{\mathrm{S}}^{i} \frac{\left(v_{\mathrm{H}_{2} \mathrm{O}}-v_{\mathrm{H}_{2}}\right) S}{2 F}(i) E_{I} \\
-c_{\mathrm{p}}^{\mathrm{H}_{2}} \hat{T}_{\mathrm{S}}^{i-1} \hat{F}_{\mathrm{A}}^{\mathrm{in}}-c_{\mathrm{p}}^{\mathrm{H}_{2}} \hat{T}_{\mathrm{S}}^{i-1} E_{F_{\mathrm{A}}^{\mathrm{in}}}-c_{\mathrm{p}}^{\mathrm{H}_{2}} T_{\mathrm{S}}^{i-1} \frac{\left(v_{\mathrm{H}_{2} \mathrm{O}}-v_{\mathrm{H}_{2}}\right) S}{2 F}(i) \hat{I}-c_{\mathrm{p}}^{\mathrm{H}_{2}} T_{\mathrm{S}}^{i-1} \frac{\left(v_{\mathrm{H}_{2} \mathrm{O}}-v_{\mathrm{H}_{2}}\right) S}{2 F}(i) E_{I}
\end{array}\right) \\
& -E_{y_{\mathrm{H}_{2} \mathrm{O}}^{i}}\left(\begin{array}{l}
c_{\mathrm{p}}^{\mathrm{H}_{2} \mathrm{O}} \hat{T}_{\mathrm{S}}^{i} \hat{F}_{\mathrm{A}}^{\mathrm{in}}+c_{\mathrm{p}}^{\mathrm{H}_{2} \mathrm{O}} \hat{T}_{\mathrm{S}}^{i} E_{F_{\mathrm{A}}^{\mathrm{in}}}+c_{\mathrm{p}}^{\mathrm{H}_{2} \mathrm{O}} T_{\mathrm{S}}^{i} \frac{\left(v_{\mathrm{H}_{2} \mathrm{O}}-v_{\mathrm{H}_{2}}\right) S}{2 F}(i) \hat{I}+c_{\mathrm{p}}^{\mathrm{H}_{2} \mathrm{O}} T_{\mathrm{S}}^{i} \frac{\left(v_{\mathrm{H}_{2} \mathrm{O}}-v_{\mathrm{H}_{2}}\right) S}{2 F}(i) E_{I} \\
-c_{\mathrm{p}}^{\mathrm{H}_{2} \mathrm{O}} \hat{T}_{\mathrm{S}}^{i-1} \hat{F}_{\mathrm{A}}^{\mathrm{in}}-c_{\mathrm{p}}^{\mathrm{H}_{2} \mathrm{O}} \hat{T}_{\mathrm{S}}^{i-1} E_{F_{\mathrm{A}}^{\mathrm{in}}}-c_{\mathrm{p}}^{\mathrm{H}_{2} \mathrm{O}} T_{\mathrm{S}}^{i-1} \frac{\left(v_{\mathrm{H}_{2} \mathrm{O}}-v_{\mathrm{H}_{2}}\right) S}{2 F}(i) \hat{I}-c_{\mathrm{p}}^{\mathrm{H}_{2} \mathrm{O}} T_{\mathrm{S}}^{i-1} \frac{\left(v_{\mathrm{H}_{2} \mathrm{O}}-v_{\mathrm{H}_{2}}\right) S}{2 F}(i) E_{I}
\end{array}\right) \\
& -E_{y_{\mathrm{O}_{2}}^{i}}\left(\begin{array}{l}
c_{\mathrm{p}}^{\mathrm{O}_{2}} \hat{T}_{\mathrm{S}}^{i} \hat{F}_{\mathrm{C}}^{\mathrm{in}}+c_{\mathrm{p}}^{\mathrm{O}_{2}} \hat{T}_{\mathrm{S}}^{i} E_{F_{\mathrm{C}}^{\mathrm{in}}}-c_{\mathrm{p}}^{\mathrm{O}_{2}} T_{\mathrm{S}}^{i} \frac{v_{\mathrm{O}_{2}} S}{2 F}(i) \hat{I}-c_{\mathrm{p}}^{\mathrm{O}_{2}} T_{\mathrm{S}}^{i} \frac{v_{\mathrm{O}_{2}} S}{2 F}(i) E_{I} \\
-c_{\mathrm{p}}^{\mathrm{O}_{2}} \hat{T}_{\mathrm{S}}^{i-1} \hat{F}_{\mathrm{C}}^{\mathrm{in}}-c_{\mathrm{p}}^{\mathrm{O}_{2}} \hat{T}_{\mathrm{S}}^{i-1} E_{F_{\mathrm{C}}^{\mathrm{in}}}+c_{\mathrm{p}}^{\mathrm{O}_{2}} T_{\mathrm{S}}^{i-1} \frac{v_{\mathrm{O}_{2}} S}{2 F}(i) \hat{I}+c_{\mathrm{p}}^{\mathrm{O}_{2}} T_{\mathrm{S}}^{i-1} \frac{v_{\mathrm{O}_{2}} S}{2 F}(i) E_{I}
\end{array}\right) \\
& -E_{F_{\mathrm{A}}}\left(\hat{y}_{\mathrm{H}_{2}}^{i} c_{\mathrm{p}}^{\mathrm{H}_{2}} \hat{T}_{\mathrm{S}}^{i}+\hat{y}_{\mathrm{H}_{2} \mathrm{O}}^{\mathrm{i}} c_{\mathrm{p}}^{\mathrm{H}_{2} \mathrm{O}} \hat{T}_{\mathrm{S}}^{i}-\hat{y}_{\mathrm{H}_{2}}^{i} c_{\mathrm{p}}^{\mathrm{H}_{2}} \hat{T}_{\mathrm{S}}^{i-1}-\hat{y}_{\mathrm{H}_{2} \mathrm{O}}^{\mathrm{i}} c_{\mathrm{p}}^{\mathrm{H}_{2} \mathrm{O}} \hat{T}_{\mathrm{S}}^{i-1}\right)-E_{F_{\mathrm{C}}}\left(\hat{y}_{\mathrm{O}_{2}}^{i} c_{\mathrm{p}}^{\mathrm{O}_{2}} \hat{T}_{\mathrm{S}}^{i-1}+y_{\mathrm{N}_{2}}^{\mathrm{i}} c_{\mathrm{p}}^{\mathrm{N}_{2}} T_{\mathrm{S}}^{i-1}\right) \\
& -E_{T_{\mathrm{S}}^{i}}\left(\begin{array}{l}
\hat{y}_{\mathrm{H}_{2}}^{i} c_{\mathrm{p}}^{\mathrm{H}_{2}} \hat{F}_{\mathrm{A}}^{\mathrm{in}}+\hat{y}_{\mathrm{H}_{2} \mathrm{O}}^{i} c_{\mathrm{p}}^{\mathrm{H}_{2} \mathrm{O}} \hat{F}_{\mathrm{A}}^{\mathrm{in}}+\hat{y}_{\mathrm{H}_{2}}^{i} c_{\mathrm{p}}^{\mathrm{H}_{2}} \frac{\left(v_{\mathrm{H}_{2} \mathrm{O}}-v_{\mathrm{H}_{2}}\right) S}{2 F}(i) \hat{I}+\hat{y}_{\mathrm{H}_{2} \mathrm{O}}^{i} c_{\mathrm{p}}^{\mathrm{H}_{2} \mathrm{O}} \frac{\left(v_{\mathrm{H}_{2} \mathrm{O}}-v_{\mathrm{H}_{2}}\right) S}{2 F}(i) \hat{I} \\
+\hat{y}_{\mathrm{O}_{2}}^{i} c_{\mathrm{p}}^{\mathrm{O}_{2}} \hat{F}_{\mathrm{C}}^{\mathrm{in}}+\hat{y}_{\mathrm{O}_{2}}^{i} c_{\mathrm{p}}^{\mathrm{O}_{2}} \frac{v_{\mathrm{O}_{2}} S}{2 F}(i) \hat{I}+y_{\mathrm{N}_{2}}^{\mathrm{in}} c_{\mathrm{p}}^{\mathrm{N}_{2}} \frac{v_{\mathrm{O}_{2}} S}{2 F}(i) \hat{I}+y_{\mathrm{N}_{2}}^{\text {in }} c_{\mathrm{p}}^{\mathrm{N}_{2}} \hat{F}_{\mathrm{C}}^{\mathrm{in}}-C_{E_{0}}^{2} \hat{I}
\end{array}\right) \\
& +\frac{\left(E_{I}+\hat{I}\right) R T_{\mathrm{S}}^{i}}{2 F} \ln \left(\frac{I_{\mathrm{L}}}{I_{\mathrm{L}}-E_{I}-\hat{I}}\right)-\frac{\hat{I} R \hat{T}_{\mathrm{S}}^{i}}{2 F} \ln \left(\frac{I_{\mathrm{L}}}{I_{\mathrm{L}}-\hat{I}}\right)+\frac{2\left(E_{I}+\hat{I}\right) T_{\mathrm{S}}^{i} R}{F} \sinh ^{-1}\left(\frac{0.5\left(E_{I}+\hat{I}\right)}{I_{0}}\right) \\
& -\frac{2 \hat{I} T_{\mathrm{S}}^{i} R}{F} \sinh ^{-1}\left(\frac{0.5 \hat{I}}{I_{0}}\right)-\frac{\left(E_{I}+\hat{I}\right) R T_{\mathrm{S}}^{i}}{2 F} \ln \left(\frac{\left(E_{y_{\mathrm{H}_{2}}^{i}}+\hat{y}_{\mathrm{H}_{2}}^{i}\right)\left(E_{y_{\mathrm{O}_{2}}^{i}}+\hat{y}_{\mathrm{O}_{2}}^{i}\right)^{0.5}}{\left(E_{\mathrm{H}_{2} \mathrm{O}}^{i}+\hat{y}_{\mathrm{H}_{2} \mathrm{O}}^{i}\right)}\right)+\frac{\hat{I} R \hat{T}_{\mathrm{S}}^{i}}{2 F} \ln \left(\frac{\left(\hat{y}_{\mathrm{H}_{2}}^{i}\right)\left(\hat{y}_{\mathrm{O}_{2}}^{i}\right)^{0.5}}{\left(\hat{y}_{\mathrm{H}_{2} \mathrm{O}}^{i}\right)}\right)
\end{aligned}
$$

Where, $i=1$ for Eq. (31) and $i=2, \cdots, 6$ for Eq. (32).

The parameter estimation errors are as follows: 


$$
\begin{aligned}
& \frac{d E_{F_{\mathrm{A}}}^{\text {in }}}{d t}=\frac{\lambda_{1}}{\rho_{\mathrm{mol}}^{\mathrm{f}} V_{\mathrm{gas}}^{\mathrm{f}}}\left(-E_{y_{\mathrm{H}_{2}}^{1}} y_{\mathrm{H}_{2}}^{\text {in }}+\hat{y}_{\mathrm{H}_{2}}^{1} E_{y_{\mathrm{H}_{2}}^{1}}-E_{y_{\mathrm{H}_{2} \mathrm{O}}^{1}} y_{\mathrm{H}_{2} \mathrm{O}}^{\text {in }}+\hat{y}_{\mathrm{H}_{2} \mathrm{O}}^{1} E_{y_{\mathrm{H}_{2} \mathrm{O}}^{1}}\right) \\
& \frac{d E_{F_{\mathrm{C}}^{\mathrm{in}}}}{d t}=\frac{\lambda_{2}}{\rho_{\mathrm{mol}}^{\mathrm{a}} V_{\mathrm{gas}}^{\mathrm{a}}}\left(-E_{y_{\mathrm{O}_{2}}^{1}} y_{\mathrm{O}_{2}}^{\text {in }}+\hat{y}_{\mathrm{O}_{2}}^{1} E_{y_{\mathrm{O}_{2}}^{\mathrm{1}}}\right) \\
& \frac{d E_{I}}{d t}=\frac{\lambda_{3}}{\rho_{\mathrm{mol}}^{\mathrm{a}} V_{\mathrm{gas}}^{\mathrm{a}}}\left(\begin{array}{l}
\hat{y}_{\mathrm{H}_{2}}^{1} E_{y_{\mathrm{H}_{2}}^{1}} \frac{\left(v_{\mathrm{H}_{2} \mathrm{O}}-v_{\mathrm{H}_{2}}\right) S}{2 F}+E_{y_{\mathrm{H}_{2}}^{1}} \frac{v_{\mathrm{H}_{2}} S}{2 F} \\
+\hat{y}_{\mathrm{H}_{2} \mathrm{O}}^{1} E_{y_{\mathrm{H}_{2} \mathrm{O}}^{1}} \frac{\left(v_{\mathrm{H}_{2} \mathrm{O}}-v_{\mathrm{H}_{2}}\right) S}{2 F}-E_{y_{\mathrm{H}_{2} \mathrm{O}}^{1}} \frac{v_{\mathrm{H}_{2} \mathrm{O}} S}{2 F}
\end{array}\right) \\
& +\frac{1}{\rho_{\mathrm{mol}}^{\mathrm{c}} V_{\mathrm{gas}}^{\mathrm{c}}}\left(-\hat{y}_{\mathrm{O}_{2}}^{1} E_{y_{\mathrm{O}_{2}}^{1}} \frac{v_{\mathrm{O}_{2}} S}{2 F}+E_{y_{\mathrm{O}_{2}}^{1}} \frac{v_{\mathrm{O}_{2}} S}{2 F}\right) \\
& \frac{d E_{T^{\text {in }}}}{d t}=-\frac{\lambda_{4}}{\rho_{\mathrm{S}} V_{\mathrm{S}} c_{\mathrm{p}}^{\mathrm{S}}}\left(\hat{F}_{\mathrm{A}}^{\text {in }}\left(y_{\mathrm{H}_{2}}^{\text {in }} c_{\mathrm{p}}^{\mathrm{H}_{2}}+y_{\mathrm{H}_{2} \mathrm{O}}^{\text {in }} c_{\mathrm{p}}^{\mathrm{H}_{2} \mathrm{O}}\right) E_{T_{\mathrm{S}}^{1}}+\hat{F}_{\mathrm{C}}^{\text {in }}\left(y_{\mathrm{O}_{2}}^{\text {in }} c_{\mathrm{p}}^{\mathrm{O}_{2}}+y_{\mathrm{N}_{2}}^{\text {in }} c_{\mathrm{p}}^{\mathrm{N}_{2}}\right) E_{T_{\mathrm{S}}^{1}}\right)
\end{aligned}
$$

The stability analysis for the proposed observer is based on the concept of input-to-state stability for cascaded systems [15]. The asymptotic stability of the system of equations $\mathbf{E}_{1}$ is proven first. The asymptotic stability of unforced system $\mathbf{E}_{2}$ with the system $\mathbf{E}_{1}$ as the input is then proven. Then, considering systems $\mathbf{E}_{1}$ and $\mathbf{E}_{2}$ as inputs, the asymptotic stability of the unforced system $\mathbf{E}_{3}$ is proved. Lastly, the stability of $\mathbf{E}_{4}$ is proven with $\mathbf{E}_{1} \mathbf{E}_{2}$ and $\mathbf{E}_{3}$ as inputs.

The error dynamics in Eqs. (1-12) are described in terms of the sets as described in Eqs. (1316) in cascaded form as:

$$
\begin{aligned}
& \dot{\mathbf{E}}_{1}=f_{1}\left(\mathbf{E}_{1}\right) \\
& \dot{\mathbf{E}}_{2}=f_{2}\left(\mathbf{E}_{1}, \mathbf{E}_{2}\right)
\end{aligned}
$$


$\dot{\mathbf{E}}_{3}=f_{2}\left(\mathbf{E}_{1}, \mathbf{E}_{2}, \mathbf{E}_{3}\right)$

$\dot{\mathbf{E}}_{4}=f_{2}\left(\mathbf{E}_{1}, \mathbf{E}_{2}, \mathbf{E}_{3}, \mathbf{E}_{4}\right)$

The global uniform asymptotic stability of the origin of the cascaded system described in Eqs.(37) and (38) is assured [15] if, the origin of Eq. (37) is globally uniformly asymptotically stable and the origin of the Eq. (38) with $\mathbf{E}_{1}$ as input is input-to-state stable. If system comprising of Eq. (39) is proved to be input-to-state stable with $\mathbf{E}_{1}$ and $\mathbf{E}_{2}$ (which are themselves asymptotically stable) as inputs, then the system comprising of Eqs. (37), (38) and (39) is globally uniformly asymptotically stable. Similarly, if the asymptotically stable system comprising of Eqs. (37), (38) and (39) are considered as inputs to the system in Eq. (40) and the origin of Eq. (40) is proved to be input-to-state stable, this means that the complete system of equation from Eq. (37-40) is globally uniformly asymptotically stable.

To prove the asymptotic stability of the system comprising Eq. (37), consider the following Lyapunov function candidate:

$V=\frac{1}{\lambda_{1}} E_{F_{\mathrm{A}}^{\mathrm{in}}}^{2}+\frac{1}{\lambda_{2}} E_{F_{\mathrm{C}}^{\mathrm{in}}}^{2}+E_{y_{\mathrm{H}_{2}}^{1}}^{2}+E_{y_{\mathrm{H}_{2} \mathrm{O}}^{1}}^{2}+E_{y_{\mathrm{O}_{2}}}^{2}+\frac{1}{\lambda_{3}} E_{I}^{2}$

It can be seen that $V=0$, when the errors are zero and positive otherwise. Therefore the Lyapunov function is positive definite. The function is also continuously differentiable and radially unbounded.

The derivative of the Lyapunov function is given as:

$$
\dot{V}=\frac{\partial V}{\partial E_{F_{\mathrm{A}}}^{\text {in }}} \dot{E}_{F_{\mathrm{A}}^{\text {in }}}+\frac{\partial V}{\partial E_{F_{\mathrm{C}}^{\mathrm{in}}}} \dot{E}_{F_{\mathrm{C}}^{\mathrm{in}}}+\frac{\partial V}{\partial E_{I}} \dot{E}_{I}+\frac{\partial V}{\partial E_{y_{\mathrm{H}_{2}}^{1}}} \dot{E}_{y_{\mathrm{H}_{2}}^{1}}+\frac{\partial V}{\partial E_{y_{\mathrm{H}_{2} \mathrm{O}}}} \dot{E}_{y_{\mathrm{H}_{2} \mathrm{O}}^{1}}+\frac{\partial V}{\partial E_{y_{\mathrm{O}_{2}}^{1}}} \dot{E}_{y_{\mathrm{O}_{2}}^{1}}
$$


which after substitution becomes:

$$
\begin{aligned}
\dot{V}= & -2 E_{y_{\mathrm{H}_{2}}^{1}}^{2}\left(F_{\mathrm{A}}^{\text {in }}-\sum_{j=1}^{i} r_{\mathrm{H}_{2}}^{j}(I)+\sum_{j=1}^{i} r_{\mathrm{H}_{2} \mathrm{O}}^{j}(I)\right)-2 E_{y_{\mathrm{H}_{2} \mathrm{O}}^{1}}^{2}\left(F_{\mathrm{A}}^{\mathrm{in}}-\sum_{j=1}^{i} r_{\mathrm{H}_{2}}^{j}(I)+\sum_{j=1}^{i} r_{\mathrm{H}_{2} \mathrm{O}}^{j}(I)\right) \\
& -2 E_{y_{\mathrm{O}_{2}}^{1}}^{2}\left(F_{\mathrm{C}}^{\mathrm{in}}-\sum_{j=1}^{i} r_{\mathrm{O}_{2}}^{j}(I)\right)
\end{aligned}
$$

It can be noted that the derivative of the Lyapunov function in the form in Eq. (43) is obtained because the formulation of the adaptation laws for the three adapted variables (viz. $\left.F_{\mathrm{A}}^{\mathrm{in}}, F_{\mathrm{C}}^{\mathrm{in}}, I\right)$ is such that the terms involving the corresponding error terms in $\dot{V}$ are cancelled. It is apparent that the derivative of the Lyapunov function is negative definite since the terms inside the brackets are positive quantities. This proves the global uniform asymptotic stability of the origin of the set of equations in Eq. (37).

Next, Eq. (38) is examined as an unforced system where the inputs from Eq. (37) are zero in order to establish its input-to-state stability. Eliminating the terms corresponding to $\mathbf{E}_{1}$ in Eqs. (38), a set of linear differential equations is obtained that can be written in matrix vector notation as follows: 


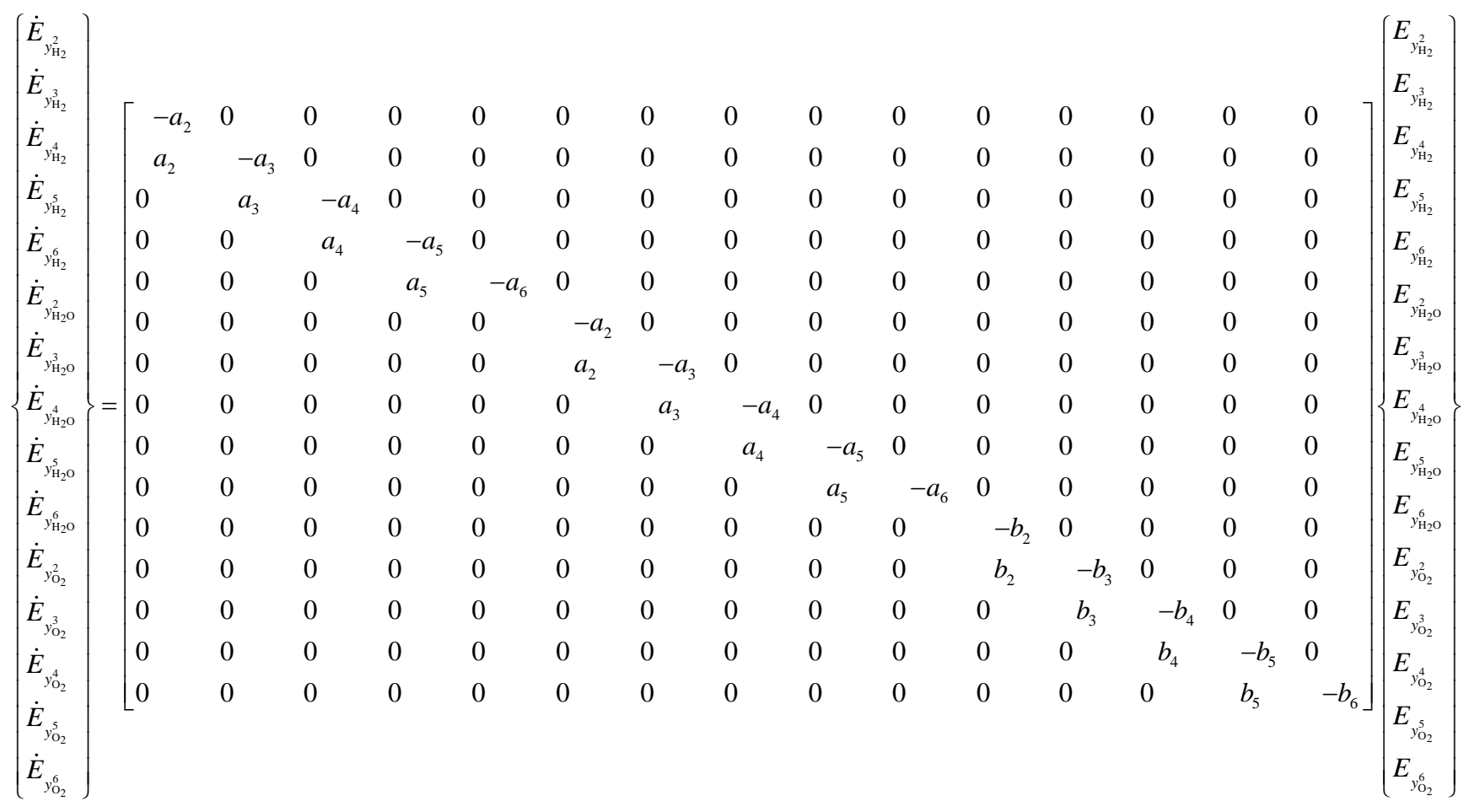

Where,

$a_{2}=F_{\mathrm{A}}^{\mathrm{in}}-\sum_{j=1}^{2} r_{\mathrm{H}_{2}}^{j}(I)+\sum_{j=1}^{2} r_{\mathrm{H}_{2} \mathrm{O}}^{j}(I)$

$a_{3}=F_{\mathrm{A}}^{\mathrm{in}}-\sum_{j=1}^{3} r_{\mathrm{H}_{2}}^{j}(I)+\sum_{j=1}^{3} r_{\mathrm{H}_{2} \mathrm{O}}^{j}(I)$

$a_{4}=F_{\mathrm{A}}^{\mathrm{in}}-\sum_{j=1}^{4} r_{\mathrm{H}_{2}}^{j}(I)+\sum_{j=1}^{4} r_{\mathrm{H}_{2} \mathrm{O}}^{j}(I)$

$a_{5}=F_{\mathrm{A}}^{\mathrm{in}}-\sum_{j=1}^{5} r_{\mathrm{H}_{2}}^{j}(I)+\sum_{j=1}^{5} r_{\mathrm{H}_{2} \mathrm{O}}^{j}(I)$ 


$$
\begin{aligned}
& a_{6}=F_{\mathrm{A}}^{\text {in }}-\sum_{j=1}^{6} r_{\mathrm{H}_{2}}^{j}(I)+\sum_{j=1}^{6} r_{\mathrm{H}_{2} \mathrm{O}}^{j}(I) \\
& b_{2}=F_{\mathrm{C}}^{\text {in }}-\sum_{j=1}^{2} r_{\mathrm{O}_{2}}^{j}(I) \\
& b_{3}=F_{\mathrm{C}}^{\text {in }}-\sum_{j=1}^{3} r_{\mathrm{O}_{2}}^{j}(I) \\
& b_{4}=F_{\mathrm{C}}^{\text {in }}-\sum_{j=1}^{4} r_{\mathrm{O}_{2}}^{j}(I) \\
& b_{5}=F_{\mathrm{C}}^{\text {in }}-\sum_{j=1}^{5} r_{\mathrm{O}_{2}}^{j}(I) \\
& b_{6}=F_{\mathrm{C}}^{\text {in }}-\sum_{j=1}^{6} r_{\mathrm{O}_{2}}^{j}(I)
\end{aligned}
$$

The constants $a_{1}, \ldots, b_{6}$ are composed of known quantities and it can be verified that the matrix in Eq. (44) has real negative eigen-values. Therefore, the unforced system described in Eq. (38) is globally exponentially stable. This proves the input-to-state stability of the origin of the system in Eq. (38) and the global asymptotic stability of the system comprising of Eqs. (37) and (38).

In the following, the input-to-state stability of the system in Eq. (39) is proven considering $\mathbf{E}_{1}$ and $\mathbf{E}_{2}$ as inputs to it. Eliminating the terms corresponding to $\mathbf{E}_{1}$ and $\mathbf{E}_{2}$ in Eqs. (39), the following differential equation is obtained for $\mathbf{E}_{3}$. 


$$
\begin{aligned}
& \rho_{\mathrm{S}} V_{\mathrm{S}} c_{\mathrm{p}}^{\mathrm{S}} \frac{d E_{T_{\mathrm{S}}^{1}}}{d t}=+\hat{F}_{\mathrm{A}}^{\text {in }} E_{T^{\text {in }}}\left(y_{\mathrm{H}_{2}}^{\text {in }} c_{\mathrm{p}}^{\mathrm{H}_{2}}+y_{\mathrm{H}_{2} \mathrm{O}}^{\text {in }} c_{\mathrm{p}}^{\mathrm{H}_{2} \mathrm{O}}\right)+\left(y_{\mathrm{O}_{2}}^{\text {in }} c_{\mathrm{p}}^{\mathrm{O}_{2}}+y_{\mathrm{N}_{2}}^{\text {in }} c_{\mathrm{p}}^{\mathrm{N}_{2}}\right) \hat{F}_{\mathrm{C}}^{\text {in }} E_{T^{\text {in }}} \\
& -E_{T_{\mathrm{S}}^{1}}\left(\begin{array}{l}
y_{\mathrm{H}_{2}}^{1} c_{\mathrm{p}}^{\mathrm{H}_{2}} F_{\mathrm{A}}^{\mathrm{in}}+y_{\mathrm{H}_{2} \mathrm{O}}^{1} c_{\mathrm{p}}^{\mathrm{H}_{2} \mathrm{O}} F_{\mathrm{A}}^{\text {in }}+y_{\mathrm{H}_{2}}^{1} c_{\mathrm{p}}^{\mathrm{H}_{2}} \frac{\left(v_{\mathrm{H}_{2} \mathrm{O}}-v_{\mathrm{H}_{2}}\right) S}{2 F} I+y_{\mathrm{H}_{2} \mathrm{O}}^{1} c_{\mathrm{p}}^{\mathrm{H}_{2} \mathrm{O}} \frac{\left(v_{\mathrm{H}_{2} \mathrm{O}}-v_{\mathrm{H}_{2}}\right) S}{2 F} I \\
+y_{\mathrm{O}_{2}}^{1} c_{\mathrm{p}}^{\mathrm{O}_{2}} F_{\mathrm{C}}^{\text {in }}+y_{\mathrm{O}_{2}}^{1} c_{\mathrm{p}}^{\mathrm{O}_{2}} \frac{v_{\mathrm{O}_{2}} S}{2 F} I+y_{\mathrm{N}_{2}}^{\text {in }} c_{\mathrm{p}}^{\mathrm{N}_{2}} \frac{v_{\mathrm{O}_{2}} S}{2 F} I+y_{\mathrm{N}_{2}}^{\text {in }} c_{\mathrm{p}}^{\mathrm{N}_{2}} F_{\mathrm{C}}^{\text {in }}
\end{array}\right)
\end{aligned}
$$

The following Lyapunov function is considered for the stability analysis.

$$
V=\frac{1}{\lambda_{4}} E_{T^{\text {in }}}^{2}+E_{T_{\mathrm{s}}^{1}}^{2}
$$

It can be seen that $V=0$, when the errors are zero and positive otherwise. Therefore the Lyapunov function is positive definite. The function is also continuously differentiable and radially unbounded.

The derivative of the Lyapunov function is given as:

$$
\dot{V}=-2 E_{\mathrm{T}_{\mathrm{S}}^{1}}^{2}\left(\begin{array}{l}
y_{\mathrm{H}_{2}}^{1} c_{\mathrm{p}}^{\mathrm{H}_{2}}\left(F_{\mathrm{A}}^{\mathrm{in}}+\frac{\left(v_{\mathrm{H}_{2} \mathrm{O}}-v_{\mathrm{H}_{2}}\right) S}{2 F} I\right)+y_{\mathrm{H}_{2} \mathrm{O}}^{1} c_{\mathrm{p}}^{\mathrm{H}_{2} \mathrm{O}}\left(F_{\mathrm{A}}^{\text {in }}+\frac{\left(v_{\mathrm{H}_{2} \mathrm{O}}-v_{\mathrm{H}_{2}}\right) S}{2 F} I\right) \\
+y_{\mathrm{O}_{2}}^{1} c_{\mathrm{p}}^{\mathrm{O}_{2}}\left(F_{\mathrm{C}}^{\mathrm{in}}+\frac{v_{\mathrm{O}_{2}} S}{2 F} I\right)+y_{\mathrm{N}_{2}}^{\mathrm{in}} c_{\mathrm{p}}^{\mathrm{N}_{2}}\left(F_{\mathrm{C}}^{\text {in }}+\frac{v_{\mathrm{O}_{2}} S}{2 F} I\right)
\end{array}\right)
$$

It can be noted that the derivative of the Lyapunov function in the form in Eq. (57) is obtained because the formulation of the adaptation laws for the adapted variables $T^{\text {in }}$ is such that the terms involving the corresponding error terms in $\dot{V}$ are cancelled. It is apparent that the derivative of the Lyapunov function is negative definite since the term inside the bracket is clearly a positive quantity. This proves the input-to-state stability of the origin of the system in Eq. (37) and the global asymptotic stability of the system comprising of Eqs. (3739). 
Finally, in the following, it is proven that the system $\mathbf{E}_{4}$ is input-to-state stable with systems $\mathbf{E}_{1}, \mathbf{E}_{2}$ and $\mathbf{E}_{3}$ considered as inputs to it. Imposing this condition, the following linear differential equations are obtained.

$$
\left.\left\{\begin{array}{l}
\dot{E}_{\tau_{s}^{2}} \\
\dot{E}_{\tau_{s}^{3}} \\
\dot{E}_{\tau_{s}^{4}} \\
\dot{E}_{\tau_{s}^{5}} \\
\dot{E}_{\tau_{s}^{6}}
\end{array}\right\}=\left[\begin{array}{lllll}
-c_{2}+d_{2} & 0 & 0 & 0 & 0 \\
c_{2} & -c_{3}+d_{3} & 0 & 0 & 0 \\
0 & c_{3} & -c_{4}+d_{4} & 0 & 0 \\
0 & 0 & c_{4} & -c_{5}+d_{5} & 0 \\
0 & 0 & 0 & c_{5} & -c_{6}+d_{6}
\end{array}\right]\right\}\left\{\begin{array}{l}
E_{T_{s}^{2}} \\
E_{T_{s}^{3}} \\
E_{T_{s}^{4}} \\
E_{T_{s}^{5}} \\
E_{T_{s}^{6}}
\end{array}\right\}
$$

where,

$$
\begin{aligned}
& c_{2}=y_{\mathrm{H}_{2}}^{2} c_{\mathrm{p}}^{\mathrm{H}_{2}}\left(F_{\mathrm{A}}^{\text {in }}+\frac{2\left(v_{\mathrm{H}_{2} \mathrm{O}}-v_{\mathrm{H}_{2}}\right) S}{2 F} I\right)+y_{\mathrm{H}_{2} \mathrm{O}}^{2} c_{\mathrm{p}}^{\mathrm{H}_{2} \mathrm{O}}\left(F_{\mathrm{A}}^{\mathrm{in}}+\frac{2\left(v_{\mathrm{H}_{2} \mathrm{O}}-v_{\mathrm{H}_{2}}\right) S}{2 F} I\right) \\
& +y_{\mathrm{O}_{2}}^{2} c_{\mathrm{p}}^{\mathrm{O}_{2}}\left(F_{\mathrm{C}}^{\text {in }}-\frac{2 v_{\mathrm{O}_{2}} S}{2 F} I\right)+y_{\mathrm{N}_{2}}^{\text {in }} c_{\mathrm{p}}^{\mathrm{N}_{2}}\left(F_{\mathrm{C}}^{\mathrm{in}}+\frac{2 v_{\mathrm{O}_{2}} S}{2 F} I\right) \\
& c_{3}=y_{\mathrm{H}_{2}}^{3} c_{\mathrm{p}}^{\mathrm{H}_{2}}\left(F_{\mathrm{A}}^{\text {in }}+\frac{3\left(v_{\mathrm{H}_{2} \mathrm{O}}-v_{\mathrm{H}_{2}}\right) S}{2 F} I\right)+y_{\mathrm{H}_{2} \mathrm{O}}^{3} c_{\mathrm{p}}^{\mathrm{H}_{2} \mathrm{O}}\left(F_{\mathrm{A}}^{\text {in }}+\frac{3\left(v_{\mathrm{H}_{2} \mathrm{O}}-v_{\mathrm{H}_{2}}\right) S}{2 F} I\right) \\
& +y_{\mathrm{O}_{2}}^{3} c_{\mathrm{p}}^{\mathrm{O}_{2}}\left(F_{\mathrm{C}}^{\text {in }}-\frac{3 v_{\mathrm{O}_{2}} S}{2 F} I\right)+y_{\mathrm{N}_{2}}^{\text {in }} c_{\mathrm{p}}^{\mathrm{N}_{2}}\left(F_{\mathrm{C}}^{\text {in }}+\frac{3 v_{\mathrm{O}_{2}} S}{2 F} I\right) \\
& c_{4}=y_{\mathrm{H}_{2}}^{4} c_{\mathrm{p}}^{\mathrm{H}}\left(F_{\mathrm{A}}^{\text {in }}+\frac{4\left(v_{\mathrm{H}_{2} \mathrm{O}}-v_{\mathrm{H}_{2}}\right) S}{2 F} I\right)+y_{\mathrm{H}_{2} \mathrm{O}}^{4} c_{\mathrm{p}}^{\mathrm{H}_{2} \mathrm{O}}\left(F_{\mathrm{A}}^{\mathrm{in}}+\frac{4\left(v_{\mathrm{H}_{2} \mathrm{O}}-v_{\mathrm{H}_{2}}\right) S}{2 F} I\right) \\
& +y_{\mathrm{O}_{2}}^{4} c_{\mathrm{p}}^{\mathrm{O}_{2}}\left(F_{\mathrm{C}}^{\text {in }}-\frac{4 v_{\mathrm{O}_{2}} S}{2 F} I\right)+y_{\mathrm{N}_{2}}^{\text {in }} c_{\mathrm{p}}^{\mathrm{N}_{2}}\left(F_{\mathrm{C}}^{\text {in }}+\frac{4 v_{\mathrm{O}_{2}} S}{2 F} I\right) \\
& c_{5}=y_{\mathrm{H}_{2}}^{5} c_{\mathrm{p}}^{\mathrm{H}_{2}}\left(F_{\mathrm{A}}^{\text {in }}+\frac{5\left(v_{\mathrm{H}_{2} \mathrm{O}}-v_{\mathrm{H}_{2}}\right) S}{2 F} I\right)+y_{\mathrm{H}_{2} \mathrm{O}}^{5} c_{\mathrm{p}}^{\mathrm{H}_{2} \mathrm{O}}\left(F_{\mathrm{A}}^{\text {in }}+\frac{5\left(v_{\mathrm{H}_{2} \mathrm{O}}-v_{\mathrm{H}_{2}}\right) S}{2 F} I\right) \\
& +y_{\mathrm{O}_{2}}^{5} c_{\mathrm{p}}^{\mathrm{O}_{2}}\left(F_{\mathrm{C}}^{\text {in }}-\frac{5 v_{\mathrm{O}_{2}} S}{2 F} I\right)+y_{\mathrm{N}_{2}}^{\text {in }} c_{\mathrm{p}}^{\mathrm{N}_{2}}\left(F_{\mathrm{C}}^{\text {in }}+\frac{5 v_{\mathrm{O}_{2}} S}{2 F} I\right)
\end{aligned}
$$




$$
\begin{aligned}
& c_{6}=y_{\mathrm{H}_{2}}^{6} c_{\mathrm{p}}^{\mathrm{H}_{2}}\left(F_{\mathrm{A}}^{\text {in }}+\frac{6\left(v_{\mathrm{H}_{2} \mathrm{O}}-v_{\mathrm{H}_{2}}\right) S}{2 F} I\right)+y_{\mathrm{H}_{2} \mathrm{O}}^{6} c_{\mathrm{p}}^{\mathrm{H}_{2} \mathrm{O}}\left(F_{\mathrm{A}}^{\text {in }}+\frac{6\left(v_{\mathrm{H}_{2} \mathrm{O}}-v_{\mathrm{H}_{2}}\right) S}{2 F} I\right) \\
& +y_{\mathrm{O}_{2}}^{6} c_{\mathrm{p}}^{\mathrm{O}_{2}}\left(F_{\mathrm{C}}^{\text {in }}-\frac{6 v_{\mathrm{O}_{2}} S}{2 F} I\right)+y_{\mathrm{N}_{2}}^{\text {in }} c_{\mathrm{p}}^{\mathrm{N}_{2}}\left(F_{\mathrm{C}}^{\mathrm{in}}+\frac{6 v_{\mathrm{O}_{2}} S}{2 F} I\right)
\end{aligned}
$$

$d_{2}=-C_{E_{0}}^{2} I-\frac{I R}{2 F} \ln \left(\frac{I_{\mathrm{L}}}{I_{\mathrm{L}}-I}\right)-\frac{2 I R}{F} \sinh ^{-1}\left(\frac{0.5 I}{I_{0}}\right)+\frac{I R}{2 F} \ln \left(\frac{\left(y_{\mathrm{H}_{2}}^{2}\right)\left(y_{\mathrm{O}_{2}}^{2}\right)^{0.5}}{\left(y_{\mathrm{H}_{2} \mathrm{O}}^{2}\right)}\right)$

$$
d_{3}=-C_{E_{0}}^{2} I-\frac{I R}{2 F} \ln \left(\frac{I_{\mathrm{L}}}{I_{\mathrm{L}}-I}\right)-\frac{2 I R}{F} \sinh ^{-1}\left(\frac{0.5 I}{I_{0}}\right)+\frac{I R}{2 F} \ln \left(\frac{\left(y_{\mathrm{H}_{2}}^{3}\right)\left(y_{\mathrm{O}_{2}}^{3}\right)^{0.5}}{\left(y_{\mathrm{H}_{2} \mathrm{O}}^{3}\right)}\right)
$$

$$
d_{4}=-C_{E_{0}}^{2} I-\frac{I R}{2 F} \ln \left(\frac{I_{\mathrm{L}}}{I_{\mathrm{L}}-I}\right)-\frac{2 I R}{F} \sinh ^{-1}\left(\frac{0.5 I}{I_{0}}\right)+\frac{I R}{2 F} \ln \left(\frac{\left(y_{\mathrm{H}_{2}}^{4}\right)\left(y_{\mathrm{O}_{2}}^{4}\right)^{0.5}}{\left(y_{\mathrm{H}_{2} \mathrm{O}}^{4}\right)}\right)
$$

$$
d_{5}=-C_{E_{0}}^{2} I-\frac{I R}{2 F} \ln \left(\frac{I_{\mathrm{L}}}{I_{\mathrm{L}}-I}\right)-\frac{2 I R}{F} \sinh ^{-1}\left(\frac{0.5 I}{I_{0}}\right)+\frac{I R}{2 F} \ln \left(\frac{\left(y_{\mathrm{H}_{2}}^{5}\right)\left(y_{\mathrm{O}_{2}}^{5}\right)^{0.5}}{\left(y_{\mathrm{H}_{2} \mathrm{O}}^{5}\right)}\right)
$$

$$
d_{6}=-C_{E_{0}}^{2} I-\frac{I R}{2 F} \ln \left(\frac{I_{\mathrm{L}}}{I_{\mathrm{L}}-I}\right)-\frac{2 I R}{F} \sinh ^{-1}\left(\frac{0.5 I}{I_{0}}\right)+\frac{I R}{2 F} \ln \left(\frac{\left(y_{\mathrm{H}_{2}}^{6}\right)\left(y_{\mathrm{O}_{2}}^{6}\right)^{0.5}}{\left(y_{\mathrm{H}_{2} \mathrm{O}}^{6}\right)}\right)
$$

All the diagonal terms in the matrix are negative and other terms are positive. It can be verified that the matrix in Eq. (58) has real negative eigen-values, making the unforced system described in Eq. (40) is globally exponentially stable. This proves the input-to-state stability of the origin of the system in Eq. (40) and the global asymptotic stability of the system comprising of Eqs. (37-40). Note that the observer design elaborated in this work is applicable for models with any number of lumps. 


\section{Simulation results and discussion}

In this section, the simulated responses for the model and the observer states subjected to step changes in the inputs, viz., the current density, the inlet temperature and the two inlet flow rates, are presented. In addition, the response of the observer inputs that are considered as the adaptation variables, are also presented. These responses correspond to the step change in the input variables at $500 \mathrm{~s}$ as follows: step change in inlet temperature from $1000 \mathrm{~K}$ to $1100 \mathrm{~K}$; step change in the current density from $600 \mathrm{~A} \mathrm{~m}^{-2}$ to $550 \mathrm{~A} \mathrm{~m}^{-2}$; step change in anode inlet flow from $0.0001 \mathrm{~mol} \mathrm{~s}^{-1}$ to 0.0002 to $\mathrm{mol} \mathrm{s}^{-1}$; step change in cathode inlet flow from $2.742 \mathrm{e}$ $3 \mathrm{~mol} \mathrm{~s}^{-1}$ to $3 \mathrm{e}-3 \mathrm{~mol} \mathrm{~s}^{-1}$. The fuel flow rate of $0.0001 \mathrm{~mol} \mathrm{~s}^{-1}$ corresponds to a value of 400 $\mathrm{ml} \mathrm{min}^{-1}$ and is adopted from [16]. A high step increase in the fuel flow rate is considered so as to demonstrate the relevance of the observer over a wide operating range and at low fuel utilisations. The fuel flow rate is especially susceptible to much fluctuation if a reformer is involved in the upstream. The operating conditions and geometrical parameters are taken from [16]. Some of the important parameters used in the simulations are given in Table 1.

The responses of the model states are represented in Figs. 2-5 in full lines and the responses of the observer states are represented in dashed lines. Only the results from nodes 2,4 and 6 are presented for clarity. It can be seen that the observer is able to track the state variables across all the six nodes. In Fig. 3, the species mole fractions are not changing after the step change because the change in inlet temperature has no effect on the species mole fractions. Similarly, as seen in Fig. 4, the change in anode inlet flow has no effect on the oxygen mole fraction. From Fig. 5, it is also evident that the change in cathode inlet flow has no effect on the species mole fractions on the anode side.

As discussed earlier, some tuning variables ( $\lambda$ 's) were introduced so as to achieve faster settling of the error variables. In general, the higher the value of the tuning variable, the faster 
is the convergence of the adaptation variables. The simulations revealed that any one the tuning parameter also had an influence on the convergence of other variables in the system. In order to understand this let us consider the following error dynamics equation:

$$
\begin{gathered}
\rho_{\mathrm{mol}}^{\mathrm{f}} V_{\mathrm{gas}}^{\mathrm{f}} \frac{d E_{y_{\mathrm{H}_{2}}^{2}}^{2}}{d t}=E_{y_{\mathrm{H}_{2}}^{1}}\left(F_{\mathrm{A}}^{\text {in }}+\frac{\left(v_{\mathrm{H}_{2} \mathrm{O}}-v_{\mathrm{H}_{2}}\right) S}{2 F} I\right)+\hat{y}_{\mathrm{H}_{2}}^{1} E_{F_{\mathrm{A}}^{\text {in }}}+\hat{y}_{\mathrm{H}_{2}}^{1} E_{I} \frac{\left(v_{\mathrm{H}_{2} \mathrm{O}}-v_{\mathrm{H}_{2}}\right) S}{2 F} \\
-E_{y_{\mathrm{H}_{2}}^{2}}\left(F_{\mathrm{A}}^{\text {in }}+\frac{\left(v_{\mathrm{H}_{2} \mathrm{O}}-v_{\mathrm{H}_{2}}\right) S}{F} I\right)-\hat{y}_{\mathrm{H}_{2}}^{2} E_{F_{\mathrm{A}}^{\text {in }}}-\hat{y}_{\mathrm{H}_{2}}^{2} E_{I} \frac{\left(v_{\mathrm{H}_{2} \mathrm{O}}-v_{\mathrm{H}_{2}}\right) S}{F}-E_{I} \frac{\left(v_{\mathrm{H}_{2}}\right) S}{F}
\end{gathered}
$$

Following the method in [17], Eq. (69) can be represented as follows.

$$
E_{y_{\mathrm{H}_{2}}^{2}}=\frac{1}{\left(\rho_{\mathrm{mol}}^{\mathrm{f}} V_{\mathrm{gas}}^{\mathrm{f}} P+F_{\mathrm{A}}^{\mathrm{in}}+\frac{\left(v_{\mathrm{H}_{2} \mathrm{O}}-v_{\mathrm{H}_{2}}\right) S}{F} I\right)}\left[\begin{array}{l}
E_{y_{\mathrm{H}_{2}}^{1}}\left(F_{\mathrm{A}}^{\text {in }}+\frac{\left(v_{\mathrm{H}_{2} \mathrm{O}}-v_{\mathrm{H}_{2}}\right) S}{2 F} I\right)+\hat{y}_{\mathrm{H}_{2}}^{1} E_{F_{\mathrm{A}}^{\text {in }}} \\
+\hat{y}_{\mathrm{H}_{2}}^{1} E_{I} \frac{\left(v_{\mathrm{H}_{2} \mathrm{O}}-v_{\mathrm{H}_{2}}\right) S}{2 F}-E_{I} \frac{\left(v_{\mathrm{H}_{2}}\right) S}{F} \\
-\hat{y}_{\mathrm{H}_{2}}^{2} E_{F_{\mathrm{A}}}-\hat{y}_{\mathrm{H}_{2}}^{2} E_{I} \frac{\left(v_{\mathrm{H}_{2} \mathrm{O}}-v_{\mathrm{H}_{2}}\right) S}{F}
\end{array}\right]
$$

where $\mathrm{P}$ is the Laplace operator. Let us assume that $E_{y_{\mathrm{H}_{2}}^{1}}$ and $E_{I}$ are inputs to Eq. (70). Taking into consideration that Eq. (70) should be equal to zero at steady state and assuming that only $E_{I}$ has not settled to zero, the equation reduces to the following.

$$
\hat{y}_{\mathrm{H}_{2}}^{1} E_{F_{\mathrm{A}}^{\text {in }}}+\hat{y}_{\mathrm{H}_{2}}^{1} E_{I} \frac{\left(v_{\mathrm{H}_{2} \mathrm{O}}-v_{\mathrm{H}_{2}}\right) S}{2 F}-E_{I} \frac{\left(v_{\mathrm{H}_{2}}\right) S}{F}-\hat{y}_{\mathrm{H}_{2}}^{2} E_{F_{\mathrm{A}}^{\text {in }}}-\hat{y}_{\mathrm{H}_{2}}^{2} E_{I} \frac{\left(v_{\mathrm{H}_{2} \mathrm{O}}-v_{\mathrm{H}_{2}}\right) S}{F}=0
$$

It is evident from Eq. (71), that under the given circumstances, it is possible that $E_{F_{\mathrm{A}}^{\text {in }}}$ has a non-zero solution and it can settle into this solution at steady state instead of the origin. 
Similar sort of arguments can be developed for other variables. However, if the inputs are in the form of a persistent excitation, parameter convergence can be guaranteed [17]. But in the fuel cell, the inputs are usually constant with possible step or ramp changes. Therefore, it can be understood that the coupled nature of the error dynamics makes it sensitive to the tuning parameters. In view of this, the tuning parameters were chosen as large as possible. Satisfactory convergence was obtained using the values for the tuning variables as: $\lambda_{3}=1 e 25 ; \lambda_{1}=\lambda_{2}=\lambda_{4}=1 e 4$. Any larger values resulted in problems in the numerical simulations. These large values for the tuning variables increased the simulation time. The simulation of the model and the observer for 1000 seconds using Matlab Simulink (Version 7.8.0 R2009a) in a personal computer with Intel Core 2 Duo processor with a speed of $3 \mathrm{GHz}$ and $1.93 \mathrm{~GB}$ of RAM took about 836 seconds in real time. Therefore, the proposed observer can be implemented in real time.

The quantities plotted in Figs. 6-9 are the estimated values of the input variables and are not the actual physical values of these variables. As can be seen form Figs. 6-9, some adapted input variables have a small deviation when step change is effected to other input. For example, in Fig. 6, the step change in current density causes a small deviation in the estimated value of cathode inlet flow (the error is in the order of 2e-9). Similarly there is an error in the anode inlet flow of the order of 9e-4. Again in Fig. 7, there are small errors in the anode and cathode inlet flows of the orders of 1e-13 and 1e-14, respectively. Also note that the errors in estimated inputs could be either positive or negative (for example, in Fig.6, the error in the anode and cathode inlet flows is positive, while they are negative in Fig. 7). However, these deviations are too small to have any considerable effect on the state variable estimation as seen in the simulations. 
As the tuning parameters influence the convergence of these estimated variables, large values for these parameters reduce the error in the input variables. However, this can also cause spikes in the estimated response as seen in Fig. 8. This seems to be a disadvantage of considering more adopted variables because less number of these variables did not result in any error [11]. Smaller values for the tuning parameters reduce the overshoot, but increase the error in the input estimation.

\section{Conclusions}

In this paper, an adaptive nonlinear estimation scheme for the simultaneous estimation of states and inputs in a planar SOFC is presented. The observer design is based on an improved lumped parameter model of the SOFC that is capable of providing the spatial profiles of temperatures and species concentrations. In this design, the inputs such as the anode and cathode flow rates, the inlet stream temperature and the cell current are considered to be unknown. These inputs variables are estimated in addition to the state variables. The stability of the proposed observer is proven using the Lyapunov function method and is based on the concept of input-to-state stability for cascaded systems. The simulations show that the developed observer can track the temperature and species concentration profiles in the planar SOFC during step changes in the input variables and can simultaneously estimate the inputs. The observer design methodology presented in this paper is applicable for models with any number of lumps.

The unknown input non-linear adaptive observer developed in this work requires fewer variables to be measured, is valid for a wide range of operating conditions and is robust to variation in the input variables because these have been considered as adaptive parameters in the observer design. This method also offers scope for extending the observer for the estimation of distributions in the entire stack. The non-linear model based observer presented 
in this work is also robust to the disturbances in inputs and measurement noises. These input disturbances can occur in the system because of the performance issues with the balance of plant components (like compressors, power electronics and heat exchangers) in the circuit. This approach will be extended in a future work for estimation of temperatures in a multi cell planar SOFC stack.

\section{References}

1. Y.C. Hsiao, J. R. Selman, Solid State Ionics 98(1-2) (1997) 33-38.

2. M. Sheng, , M. Mangold, A. Kienle, Journal of Power Sources 162 (2006) 1213-1219.

3. M. Dallig, J. Karl, Energy Procedia 28 (2012) 37 - 47.

4. P. Vijay, S. Hosseini, M.O. Tadé, Chemical Engineering Research and Design 91 (2013) 560-572.

5. M. Mangold, M. Krasnyk, K. Sundmacher, Journal of Applied Electrochemistry 36 (3) (2006) 265-275.

6. M. Grotsch, M. Gundermann, M. Mangold, A. Kienle, K. Sundmacher, Journal of Process Control 16 (2006) 985-992.

7. M. Mangold, M. Sheng, P. Heidebrecht, A. Kienle, K. Sundmacher, Chemical Engineering Science 59 (2004) 4847 - 4852.

8. H. Xi, J. Sun, J. Chen, Proc. of ASME International Mechanical Engineering Congress and Exposition (2007), Seattle, Washington, USA.

9. W. B. Guan, H. J. Zhai, L. Jin, C. Xu, andW. G.Wang, Fuel Cells (2012) 12(1) 24-31.

10. N. Boizot, E. Busvelle, Adaptive-Gain Observers and Applications, chapter from book, Nonlinear Observers and Applications, Lecture Notes in Control and Information Sciences, 363 (2007) 71-114. 
11. P. Vijay, M.O. Tadé, Journal of Process Control (2013) 23(3) 429-443.

12. H.A. Liebhafsky, E.J. Cairns, Fuel Cells and Fuel Batteries: A guide to their research and development, John Wiley and Sons, New York, 1968.

13. S.C. Singhal, K. Kendall, High Temperature Solid Oxide Fuel Cells: Fundamentals, Design and Applications, Elsevier Ltd., 2003.

14. P. Vijay, M.O. Tade, R. Datta, Ind. Eng. Chem. Res. 50 (2011) 1439-1452.

15. H.K. Khalil, Nonlinear systems, Second edition,Prentice Hall New Jersey, 1996.

16. F. Ravussin, J.V. Herle, N. Autissier, M. Molinelli, D. Larrain, D. Favrat, Journal of the European Ceramic Society 27 (2007) 1035-1040.

17. J. E. Slotine, W. Li, Applied nonlinear control, Prentice-Hall inc., New Jersey, 1991.

18. AKM M. Murshed, B. Huang, K. Nandakumar, Journal of Power Sources 163 (2007) $830-845$.

19. P. Vijay, A.K. Samantaray, A. Mukherjee, Mechatronics 19 (2009) 489-502.

20. Y. Qi, B. Huang, KT. Chuang, Journal of Power Sources 150 (2005) 32-47.

\section{Notations}

E $\quad$ Error

F $\quad$ Mole flow rate $\left(\mathrm{mol} \mathrm{s}^{-1}\right)$

$c_{\mathrm{p}} \quad$ Specific heat capacity $\left(\mathrm{J} \mathrm{mol}^{-1} \mathrm{~K}^{-1}\right)$

$C_{E_{0}}^{1} \quad$ Empirical constant involved in open circuit voltage calculation $(1.2586 \mathrm{~V})$ 


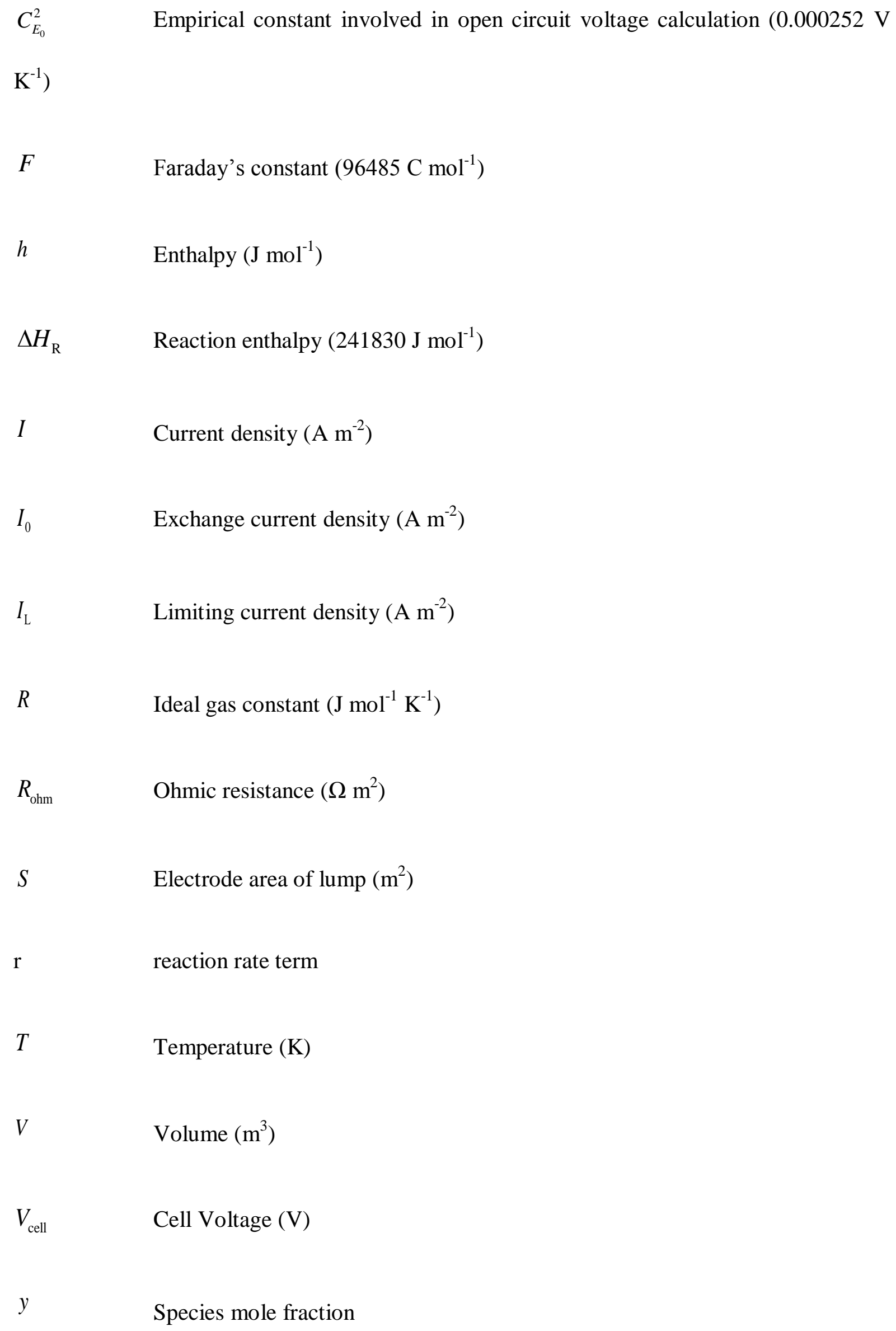




\section{Greek letters}

$\begin{array}{ll}\rho_{\mathrm{mol}} & \text { Molar Density }\left(\mathrm{mol} \mathrm{m}^{-3}\right) \\ \rho_{\mathrm{S}} c_{\mathrm{p}}^{\mathrm{S}} & 106\left(\mathrm{Jm}^{-3} \mathrm{~K}^{-1}\right) \\ v & \text { Stoichiometric coefficient } \\ \lambda & \\ & \text { Tuning parameter }\end{array}$

\section{Sub/superscripts}

in inlet

$i \quad i^{\text {th }}$ lump
A Anode
C Cathode
f Fuel
$a \quad$ Air
S Solid
out Outlet

\section{Figure captions}

Fig. 1. Schematic showing the construction and operation of the SOFC (adopted from [14]). 
Fig. 2. Observer tracking during step change in current density.

Fig. 3. Observer tracking during step change in inlet temperature.

Fig. 4. Observer tracking during step change in anode inlet flow.

Fig. 5. Observer tracking during step change in cathode inlet flow.

Fig. 6. The reaction of the adapted parameters to the step changes in inlet current density.

Fig. 7. The reaction of the adapted parameters to the step changes in inlet temperature.

Fig. 8. The reaction of the adapted parameters to the step changes in anode inlet flow.

Fig. 9. The reaction of the adapted parameters to the step changes in cathode inlet flow.

\section{Appendix}

The model development for the purpose of observer design is outlined in this appendix. The hydrogen balance for the anode channel of the SOFC results in the following set of equations.

$$
\begin{aligned}
& \rho_{\mathrm{mol}}^{\mathrm{f}} V_{\mathrm{gas}}^{\mathrm{f}} \frac{d y_{\mathrm{H}_{2}}^{i}}{d t}=y_{\mathrm{H}_{2}}^{\mathrm{in}} F_{\mathrm{A}}^{\mathrm{in}}-y_{\mathrm{H}_{2}}^{i} F_{\mathrm{A}}^{i}-r_{\mathrm{H}_{2}}^{i}(I) \\
& \rho_{\mathrm{mol}}^{\mathrm{f}} V_{\text {gas }}^{\mathrm{f}} \frac{d y_{\mathrm{H}_{2}}^{i}}{d t}=y_{\mathrm{H}_{2}}^{i-1} F_{\mathrm{A}}^{i-1}-y_{\mathrm{H}_{2}}^{i} F_{\mathrm{A}}^{i}-r_{\mathrm{H}_{2}}^{i}(I)
\end{aligned}
$$

Where, $i=1$ for Eq. (A1) and $i=2, \cdots, 6$ for Eq. (A2). 
Similarly, the water vapour balance for the anode gas channel is given by the following set of equations.

$\rho_{\text {mol }}^{\mathrm{f}} V_{\text {gas }}^{\mathrm{f}} \frac{d y_{\mathrm{H}_{2} \mathrm{O}}^{i}}{d t}=y_{\mathrm{H}_{2} \mathrm{O}}^{\text {in }} F_{\mathrm{A}}^{\text {in }}-y_{\mathrm{H}_{2} \mathrm{O}}^{i} F_{\mathrm{A}}^{i}+r_{\mathrm{H}_{2} \mathrm{O}}^{i}(I)$

$\rho_{\mathrm{mol}}^{\mathrm{f}} V_{\mathrm{gas}}^{\mathrm{f}} \frac{d y_{\mathrm{H}_{2} \mathrm{O}}^{i}}{d t}=y_{\mathrm{H}_{2} \mathrm{O}}^{i-1} F_{\mathrm{A}}^{i-1}-y_{\mathrm{H}_{2} \mathrm{O}}^{i} F_{\mathrm{A}}^{i}+r_{\mathrm{H}_{2} \mathrm{O}}^{i}(I)$

Where, $i=1$ for Eq. (A3) and $i=2, \cdots, 6$ for Eq. (A4).

The oxygen balance in the cathode channels of the SOFC are written as follows:

$\rho_{\mathrm{mol}}^{\mathrm{a}} V_{\mathrm{gas}}^{\mathrm{a}} \frac{d y_{\mathrm{O}_{2}}^{i}}{d t}=y_{\mathrm{O}_{2}}^{\mathrm{in}} F_{\mathrm{C}}^{\mathrm{in}}-y_{\mathrm{O}_{2}}^{i} F_{\mathrm{C}}^{i}-r_{\mathrm{O}_{2}}^{i}(I)$

$\rho_{\mathrm{mol}}^{\mathrm{a}} V_{\mathrm{gas}}^{\mathrm{a}} \frac{d y_{\mathrm{O}_{2}}^{i}}{d t}=y_{\mathrm{O}_{2}}^{i-1} F_{\mathrm{C}}^{i-1}-y_{\mathrm{O}_{2}}^{i} F_{\mathrm{C}}^{i}-r_{\mathrm{O}_{2}}^{i}(I)$

Where, $i=1$ for Eq. (A5) and $i=2, \cdots, 6$ for Eq. (A6).

The thermal balances for the solid volume of the SOFC are given in the following equations:

$$
\begin{aligned}
& \rho_{\mathrm{S}} V_{\mathrm{S}} c_{\mathrm{p}}^{\mathrm{s}} \frac{d T_{\mathrm{S}}^{i}}{d t}=h_{\mathrm{A}}^{\text {in }} F_{\mathrm{A}}^{\text {in }}+h_{\mathrm{C}}^{\text {in }} F_{\mathrm{C}}^{\text {in }}-h_{\mathrm{A}}^{i} F_{\mathrm{A}}^{i}-h_{\mathrm{C}}^{i} F_{\mathrm{C}}^{i}+\Delta H_{\mathrm{R}} r_{\mathrm{H}_{2}}^{i}(I)-V_{\text {cell }}^{i} I \\
& \rho_{\mathrm{S}} V_{\mathrm{S}} c_{\mathrm{p}}^{\mathrm{S}} \frac{d T_{\mathrm{S}}^{i}}{d t}=h_{\mathrm{A}}^{i-1} F_{\mathrm{A}}^{i-1}+h_{\mathrm{C}}^{i-1} F_{\mathrm{C}}^{i-1}-h_{\mathrm{A}}^{i} F_{\mathrm{A}}^{i}-h_{\mathrm{C}}^{i} F_{\mathrm{C}}^{i}+\Delta H_{\mathrm{R}} r_{\mathrm{H}_{2}}^{i}(I)-V_{\text {cell }}^{i} I
\end{aligned}
$$

Where, $i=1$ for Eq. (A7) and $i=2, \cdots, 6$ for Eq. (A8). 
The reaction rates in the preceding equations are given as:

$r_{\mathrm{H}_{2}}^{i}(I)=\frac{v_{\mathrm{H}_{2}} I}{2 F}$

$r_{\mathrm{O}_{2}}^{i}(I)=\frac{v_{\mathrm{O}_{2}} I}{2 F}$

$r_{\mathrm{H}_{2} \mathrm{O}}^{i}(I)=\frac{\nu_{\mathrm{H}_{2} \mathrm{O}} I}{2 F}$

The flows in the anode and cathode channels are given as follows:

$$
\begin{aligned}
& F_{\mathrm{A}}^{i}=F_{\mathrm{A}}^{\mathrm{in}}-\sum_{j=1}^{i} r_{\mathrm{H}_{2}}^{j}(I)+\sum_{j=1}^{i} r_{\mathrm{H}_{2} \mathrm{O}}^{j}(I) \\
& F_{\mathrm{C}}^{i}=F_{\mathrm{C}}^{\mathrm{in}}-\sum_{j=1}^{i} r_{\mathrm{O}_{2}}^{j}(I)
\end{aligned}
$$

The cell voltages in each of the lumps are given by,

$$
V_{\text {cell }}^{i}=V_{\text {ocv }}^{i}-I R_{\mathrm{ohm}}-\frac{R T_{\mathrm{S}}^{i}}{2 F} \ln \left(\frac{I_{\mathrm{L}}}{I_{\mathrm{L}}-I}\right)-\frac{2 R T_{\mathrm{S}}^{i}}{F} \sinh ^{-1}\left(\frac{0.5 I}{I_{0}}\right)
$$

where the open circuit voltage is given by the Nernst equation as:

$$
V_{\mathrm{ocV}}^{i}=E_{0}^{i}+\frac{R T_{\mathrm{S}}^{i}}{2 F} \ln \left(\frac{y_{\mathrm{H}_{2}}^{i}\left(y_{\mathrm{O}_{2}}^{i}\right)^{0.5}}{y_{\mathrm{H}_{2} \mathrm{O}}^{i}}\right)
$$

In Eq. (A15), $E_{0}$ is the standard cell potential given as [19]: 
$E_{0}^{i}=C_{E_{0}}^{1}-C_{E_{0}}^{2} T_{\mathrm{S}}^{i}$

The second, third and the fourth terms on the right hand side of Eq. (A14) represent the ohmic, concentration and the activation over-potentials [19] that are the sources of potential losses in the fuel cell. The simplified expression for the concentration over-potential involves a limiting current and is taken from $[19,20]$. The expression for the activation over-potential is obtained from the Butler-Volmer equation if the transfer co-efficients $(\alpha)$ are 0.5 , which is usually the case $[20,21]$. The ohmic resistance $\left(R_{\mathrm{ohm}}\right)$ and the exchange current $\left(I_{0}\right)$ are considered constants in this work.

The specific enthalpies in Eqs. (A7) and (A8) are given by,

$$
\begin{aligned}
& h_{\mathrm{A}}^{\text {in }}=c_{\mathrm{p}}^{\mathrm{A}} T^{\mathrm{in}}=\left(y_{\mathrm{H}_{2}}^{\text {in }} c_{\mathrm{p}}^{\mathrm{H}_{2}}+y_{\mathrm{H}_{2} \mathrm{O}}^{\text {in }} c_{\mathrm{p}}^{\mathrm{H}_{2} \mathrm{O}}\right) T^{\mathrm{in}} \\
& h_{\mathrm{A}}^{i}=c_{\mathrm{p}}^{\mathrm{A}} T_{\mathrm{S}}^{i}=\left(y_{\mathrm{H}_{2}}^{i} c_{\mathrm{p}}^{\mathrm{H}_{2}}+y_{\mathrm{H}_{2} \mathrm{O}}^{i} c_{\mathrm{p}}^{\mathrm{H}_{2} \mathrm{O}}\right) T_{\mathrm{S}}^{i} \\
& h_{\mathrm{C}}^{\text {in }}=c_{\mathrm{p}}^{\mathrm{C}} T^{\mathrm{in}}=\left(y_{\mathrm{O}_{2}}^{\text {in }} c_{\mathrm{p}}^{\mathrm{O}_{2}}+y_{\mathrm{N}_{2}}^{\text {in }} c_{\mathrm{p}}^{\mathrm{N}_{2}}\right) T^{\mathrm{in}} \\
& h_{\mathrm{C}}^{i}=c_{\mathrm{p}}^{\mathrm{C}} T_{\mathrm{S}}^{i}=\left(y_{\mathrm{O}_{2}}^{i} c_{\mathrm{p}}^{\mathrm{O}_{2}}+y_{\mathrm{N}_{2}}^{\text {in }} c_{\mathrm{p}}^{\mathrm{N}_{2}}\right) T_{\mathrm{S}}^{i}
\end{aligned}
$$

Substitution of the Eqs. (A9-A20) in Eqs. (A1-A8) results in the following set of equations for the SOFC.

$$
\rho_{\mathrm{mol}}^{\mathrm{f}} V_{\mathrm{gas}}^{\mathrm{f}} \frac{d y_{\mathrm{H}_{2}}^{i}}{d t}=y_{\mathrm{H}_{2}}^{\mathrm{in}} F_{\mathrm{A}}^{\mathrm{in}}-y_{\mathrm{H}_{2}}^{i} F_{\mathrm{A}}^{\mathrm{in}}-y_{\mathrm{H}_{2}}^{i}\left(-\sum_{j=1}^{i} r_{\mathrm{H}_{2}}^{j}(I)+\sum_{j=1}^{i} r_{\mathrm{H}_{2} \mathrm{O}}^{j}(I)\right)-r_{\mathrm{H}_{2}}^{i}(I)
$$




$$
\begin{aligned}
\rho_{\text {mol }}^{\mathrm{f}} V_{\text {gas }}^{\mathrm{f}} \frac{d y_{\mathrm{H}_{2}}^{i}}{d t}= & y_{\mathrm{H}_{2}}^{i-1} F_{\mathrm{A}}^{\mathrm{in}}+y_{\mathrm{H}_{2}}^{i-1}\left(-\sum_{j=1}^{i-1} r_{\mathrm{H}_{2}}^{j}(I)+\sum_{j=1}^{i-1} r_{\mathrm{H}_{2} \mathrm{O}}^{j}(I)\right)-y_{\mathrm{H}_{2}}^{i} F_{\mathrm{A}}^{\text {in }} \\
& -y_{\mathrm{H}_{2}}^{i}\left(-\sum_{j=1}^{i} r_{\mathrm{H}_{2}}^{j}(I)+\sum_{j=1}^{i} r_{\mathrm{H}_{2} \mathrm{O}}^{j}(I)\right)-r_{\mathrm{H}_{2}}^{i}(I)
\end{aligned}
$$

Where, $i=1$ for Eq. (A21) and $i=2, \cdots, 6$ for Eq. (A22).

Similarly, the water vapour balance for the anode gas channel is given by the following set of equations.

$$
\begin{aligned}
& \rho_{\text {mol }}^{\mathrm{f}} V_{\mathrm{gas}}^{\mathrm{f}} \frac{d y_{\mathrm{H}_{2} \mathrm{O}}^{i}}{d t}=y_{\mathrm{H}_{2} \mathrm{O}}^{\text {in }} F_{\mathrm{A}}^{\text {in }}-y_{\mathrm{H}_{2} \mathrm{O}}^{i} F_{\mathrm{A}}^{\text {in }}-y_{\mathrm{H}_{2} \mathrm{O}}^{i}\left(-\sum_{j=1}^{i} r_{\mathrm{H}_{2}}^{j}(I)+\sum_{j=1}^{i} r_{\mathrm{H}_{2} \mathrm{O}}^{j}(I)\right)+r_{\mathrm{H}_{2} \mathrm{O}}^{i}(I) \\
& \rho_{\mathrm{mol}}^{\mathrm{f}} V_{\mathrm{gas}}^{\mathrm{f}} \frac{d y_{\mathrm{H}_{2} \mathrm{O}}^{i}}{d t}=y_{\mathrm{H}_{2} \mathrm{O}}^{i-1} F_{\mathrm{A}}^{\mathrm{in}}+y_{\mathrm{H}_{2} \mathrm{O}}^{i-1}\left(-\sum_{j=1}^{i-1} r_{\mathrm{H}_{2}}^{j}(I)+\sum_{j=1}^{i-1} r_{\mathrm{H}_{2} \mathrm{O}}^{j}(I)\right)-y_{\mathrm{H}_{2} \mathrm{O}}^{i} F_{\mathrm{A}}^{\text {in }} \\
& -y_{\mathrm{H}_{2} \mathrm{O}}^{i}\left(-\sum_{j=1}^{i} r_{\mathrm{H}_{2}}^{j}(I)+\sum_{j=1}^{i} r_{\mathrm{H}_{2} \mathrm{O}}^{j}(I)\right)+r_{\mathrm{H}_{2} \mathrm{O}}^{i}(I)
\end{aligned}
$$

Where, $i=1$ for Eq. (A23) and $i=2, \cdots, 6$ for Eq. (A24).

The oxygen balance in the cathode channels of the SOFC is given as:

$$
\begin{aligned}
& \rho_{\text {mol }}^{\mathrm{a}} V_{\mathrm{gas}}^{\mathrm{a}} \frac{d y_{\mathrm{O}_{2}}^{i}}{d t}=y_{\mathrm{O}_{2}}^{\mathrm{in}} F_{\mathrm{C}}^{\mathrm{in}}-y_{\mathrm{O}_{2}}^{i} F_{\mathrm{C}}^{\mathrm{in}}-y_{\mathrm{O}_{2}}^{i}\left(-\sum_{j=1}^{i} r_{\mathrm{O}_{2}}^{j}(I)\right)-r_{\mathrm{O}_{2}}^{i}(I) \\
& \rho_{\text {mol }}^{\mathrm{a}} V_{\mathrm{gas}}^{\mathrm{a}} \frac{d y_{\mathrm{O}_{2}}^{i}}{d t}=y_{\mathrm{O}_{2}}^{i-1} F_{\mathrm{C}}^{\text {in }}+y_{\mathrm{O}_{2}}^{i-1}\left(-\sum_{j=1}^{i-1} r_{\mathrm{O}_{2}}^{j}(I)\right)-y_{\mathrm{O}_{2}}^{i} F_{\mathrm{C}}^{\mathrm{in}}-y_{\mathrm{O}_{2}}^{i}\left(-\sum_{j=1}^{i} r_{\mathrm{O}_{2}}^{j}(I)\right)-r_{\mathrm{O}_{2}}^{i}(I)
\end{aligned}
$$

Where, $i=1$ for Eq. (A25) and $i=2, \cdots, 6$ for Eq. (A26).

The thermal balances for the solid volume of the SOFC are: 


$$
\begin{aligned}
\rho_{\mathrm{S}} V_{\mathrm{S}} c_{\mathrm{p}}^{\mathrm{S}} \frac{d T_{\mathrm{S}}^{i}}{d t}= & \left(y_{\mathrm{H}_{2}}^{\mathrm{in}} c_{\mathrm{p}}^{\mathrm{H}_{2}}+y_{\mathrm{H}_{2} \mathrm{O}}^{\mathrm{in}} c_{\mathrm{p}}^{\mathrm{H}_{2} \mathrm{O}}\right) T^{\mathrm{in}} F_{\mathrm{A}}^{\mathrm{in}}+\left(y_{\mathrm{O}_{2}}^{\mathrm{in}} c_{\mathrm{p}}^{\mathrm{O}_{2}}+y_{\mathrm{N}_{2}}^{\mathrm{in}} c_{\mathrm{p}}^{\mathrm{N}_{2}}\right) T^{\mathrm{in}} F_{\mathrm{C}}^{\mathrm{in}}-\left(y_{\mathrm{H}_{2}}^{i} c_{\mathrm{p}}^{\mathrm{H}_{2}}+y_{\mathrm{H}_{2} \mathrm{O}}^{i} c_{\mathrm{p}}^{\mathrm{H}_{2} \mathrm{O}}\right) T_{\mathrm{S}}^{i} F_{\mathrm{A}}^{\mathrm{in}} \\
& -\left(y_{\mathrm{H}_{2}}^{i} c_{\mathrm{p}}^{\mathrm{H}_{2}}+y_{\mathrm{H}_{2} \mathrm{O}}^{i} c_{\mathrm{p}}^{\mathrm{H}_{2} \mathrm{O}}\right) T_{\mathrm{S}}^{i}\left(-\sum_{j=1}^{i} r_{\mathrm{H}_{2}}^{j}(I)+\sum_{j=1}^{i} r_{\mathrm{H}_{2} \mathrm{O}}^{j}(I)\right) \\
& -\left(y_{\mathrm{O}_{2}}^{i} c_{\mathrm{p}}^{\mathrm{O}_{2}}+y_{\mathrm{N}_{2}}^{\mathrm{in}} c_{\mathrm{p}}^{\mathrm{N}_{2}}\right) T_{\mathrm{S}}^{i} F_{\mathrm{C}}^{\mathrm{in}}-\left(y_{\mathrm{O}_{2}}^{i} c_{\mathrm{p}}^{\mathrm{O}_{2}}+y_{\mathrm{N}_{2}}^{\mathrm{in}} c_{\mathrm{p}}^{\mathrm{N}_{2}}\right) T_{\mathrm{S}}^{i}\left(-\sum_{j=1}^{i} r_{\mathrm{O}_{2}}^{j}(I)\right)+\frac{\Delta H_{\mathrm{R}} I}{2 F} \\
& -I\left(C_{E_{0}}^{1}-C_{E_{0}}^{2} T_{\mathrm{S}}^{i}+\frac{R T_{\mathrm{S}}^{i}}{2 F} \ln \left(\frac{y_{\mathrm{H}_{2}}^{i}\left(y_{\mathrm{O}_{2}}^{i}\right)^{0.5}}{y_{\mathrm{H}_{2} \mathrm{O}}^{i}}\right)\right) \\
& -I\left(-I R_{\mathrm{ohm}}-\frac{R T_{\mathrm{S}}^{i}}{2 F} \ln \left(\frac{I_{\mathrm{L}}}{I_{\mathrm{L}}-I}\right)-\frac{2 R T_{\mathrm{S}}^{i}}{F} \sinh ^{-1}\left(\frac{0.5 I}{I_{0}}\right)\right)
\end{aligned}
$$

$$
\begin{aligned}
\rho_{\mathrm{S}} V_{\mathrm{S}} c_{\mathrm{p}}^{\mathrm{S}} \frac{d T_{\mathrm{S}}^{i}}{d t}= & \left(y_{\mathrm{H}_{2}}^{i} c_{\mathrm{p}}^{\mathrm{H}_{2}}+y_{\mathrm{H}_{2} \mathrm{O}}^{i} c_{\mathrm{p}}^{\mathrm{H}_{2} \mathrm{O}}\right) T_{\mathrm{S}}^{i-1} F_{\mathrm{A}}^{\mathrm{in}}+\left(y_{\mathrm{H}_{2}}^{i} c_{\mathrm{p}}^{\mathrm{H}_{2}}+y_{\mathrm{H}_{2} \mathrm{O}}^{i} c_{\mathrm{p}}^{\mathrm{H}_{2} \mathrm{O}}\right) T_{\mathrm{S}}^{i-1}\left(-\sum_{j=1}^{i-1} r_{\mathrm{H}_{2}}^{j}(I)+\sum_{j=1}^{i-1} r_{\mathrm{H}_{2} \mathrm{O}}^{j}(I)\right) \\
& +\left(y_{\mathrm{O}_{2}}^{i} c_{\mathrm{p}}^{\mathrm{O}_{2}}+y_{\mathrm{N}_{2}}^{\mathrm{in}} c_{\mathrm{p}}^{\mathrm{N}_{2}}\right) T_{\mathrm{S}}^{i-1} F_{\mathrm{C}}^{\mathrm{in}}+\left(y_{\mathrm{O}_{2}}^{i} c_{\mathrm{p}}^{\mathrm{O}_{2}}+y_{\mathrm{N}_{2}}^{\mathrm{in}} c_{\mathrm{p}}^{\mathrm{N}_{2}}\right) T_{\mathrm{S}}^{i-1}\left(-\sum_{j=1}^{i-1} r_{\mathrm{O}_{2}}^{j}(I)\right) \\
& -\left(y_{\mathrm{H}_{2}}^{i} c_{\mathrm{p}}^{\mathrm{H}_{2}}+y_{\mathrm{H}_{2} \mathrm{O}}^{i} c_{\mathrm{p}}^{\mathrm{H}_{2} \mathrm{O}}\right) T_{\mathrm{S}}^{i} F_{\mathrm{A}}^{\mathrm{in}}-\left(y_{\mathrm{O}_{2}}^{i} c_{\mathrm{p}}^{\mathrm{O}_{2}}+y_{\mathrm{N}_{2}}^{\mathrm{in}} c_{\mathrm{p}}^{\mathrm{N}_{2}}\right) T_{\mathrm{S}}^{i} F_{\mathrm{C}}^{\mathrm{in}} \\
& -\left(y_{\mathrm{H}_{2}}^{i} c_{\mathrm{p}}^{\mathrm{H}_{2}}+y_{\mathrm{H}_{2} \mathrm{O}}^{i} c_{\mathrm{p}}^{\mathrm{H}_{2} \mathrm{O}}\right) T_{\mathrm{S}}^{i}\left(-\sum_{j=1}^{i} r_{\mathrm{H}_{2}}^{j}(I)+\sum_{j=1}^{i} r_{\mathrm{H}_{2} \mathrm{O}}^{j}(I)\right) \\
& -\left(y_{\mathrm{O}_{2}}^{i} c_{\mathrm{p}}^{\mathrm{O}_{2}}+y_{\mathrm{N}_{2}}^{\mathrm{in}} c_{\mathrm{p}}^{\mathrm{N}_{2}}\right) T_{\mathrm{S}}^{i}\left(-\sum_{j=1}^{i} r_{\mathrm{O}_{2}}^{j}(I)\right)+\frac{\Delta H_{\mathrm{R}} I}{2 F} \\
& -I\left(C_{E_{0}}^{1}-C_{E_{0}}^{2} T_{\mathrm{S}}^{i}+\frac{R T_{\mathrm{S}}^{i}}{2 F} \ln \left(\frac{y_{\mathrm{H}_{2}}^{i}\left(y_{\mathrm{O}_{2}}^{i}\right)^{0.5}}{y_{\mathrm{H}_{2} \mathrm{O}}^{i}}\right)\right) \\
& -I\left(-I R_{\mathrm{ohm}}-\frac{R T_{\mathrm{S}}^{i}}{2 F} \ln \left(\frac{I_{\mathrm{L}}}{I_{\mathrm{L}}-I}\right)-\frac{2 R T_{\mathrm{S}}^{i}}{F} \sinh ^{-1}\left(\frac{0.5 I}{I_{0}}\right)\right)
\end{aligned}
$$

Where, $i=1$ for Eq. (A27) and $i=2, \cdots, 6$ for Eq. (A28). The Eqs. (A21-A28) represent the SOFC lumped parameter model used for adaptive observer design. 